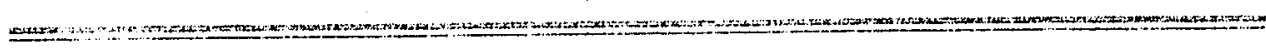

ON THE

TRANSFORMATION GROUP

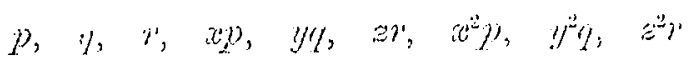

$-B Y-$

HILIARY I. RORERTS, M. A.

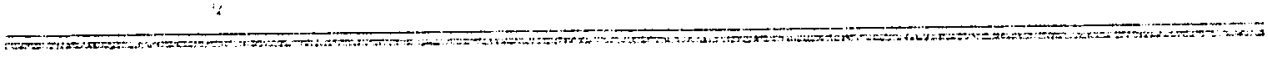

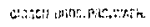


Lis

U. Va. Doctoral

Dissertation

15

75138

\section{ON THE TRANSFORMATION GROUP}

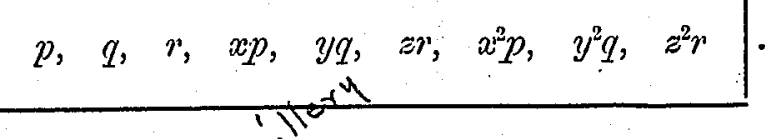

By Ë.'工. RoberTs, M. A.

"1

I.

Geometrioal Peculiarities.

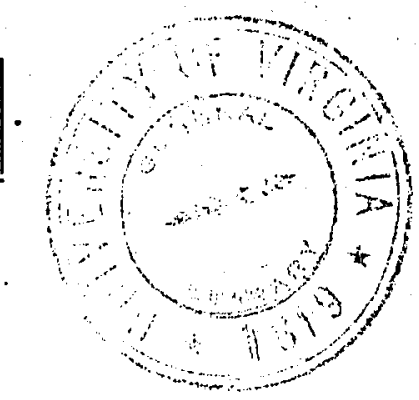

1. In this section we shall find the finite transformations and the pathcurves of the group

$$
p, \quad q, \quad r, \quad x p, \quad ? q, \quad z r, \quad x^{2} p, \quad y^{2} q, \quad z^{2} r
$$

where $p \equiv \frac{\partial f}{\partial x}, q=\frac{\partial f}{\partial y}, r=\frac{\partial f}{\partial z}$.

We shall also briefly discuss some of the geometrical peculiarities of the Group.

2. The most general infinitesimal transformation of the Group is

$$
\nabla f \equiv\left(a_{1}+2 b_{1} x+c_{1} x^{2}\right) p+\left(a_{2}+2 b_{2} y+c_{2} y^{2}\right) q+\left(a_{3}+2 b_{3} z+c_{3} z^{2}\right) r
$$

where $a_{i}, b_{i}, c_{i}(i=1,2,3)$ are certain undetermined constants.

The finite transformations are given, in the usual manner, by the integration of the following simultaneous system :

$$
\frac{d x_{1}}{a_{1}+2 b_{1} x_{1}+c_{1} x_{1}^{2}}=\frac{d y_{1}}{a_{2}+2 b_{2} y_{1}+c_{2} y_{1}^{2}}=\frac{d z_{1}}{a_{3}+2 b_{3} z_{1}+c_{3} z_{1}^{2}}=d t
$$

with the condition that $x_{1}=x, y_{1}=y, z_{1}=z$, when $t=0$.

Now, since the variables have already been separated in these equations, it is easily seen that whether $a_{i} c_{i}<=>b_{i}^{2}(i=1,2,3)$ the finite transformations have the form

$$
x_{1}=\frac{\alpha_{1} x+\alpha_{2}}{\alpha_{3} x+1}, \quad y_{1}=\frac{\beta_{1} x+\beta_{2}}{\beta_{3} x+1}, \quad z_{1}=\frac{\gamma_{1} z+\gamma_{2}}{\gamma_{3} z+1},
$$

where $\alpha_{i}, \beta_{i}, \gamma_{i}$ are certain constants. 
3. It is evident that these transformations will transform the equations

into

$$
x=\text { const., } y=\text { const., } z=\text { const. }
$$

$$
x_{1}=\text { const., } \quad y_{1}=\text { const., } \quad z_{1}=\text { const. }
$$

Hence, we observe that all the planes parallel to the yz-plane are moved parallel to themselves by the transformations of the $G_{0}$. In this case, we say these $\infty^{1}$ parallel planes form an invariant family of planes; that is, the farity is invariant as a whole, while the planes of the family are interchanged among each other by means of the transformations of the $G_{9}$.

Similarly the planes parallel to the $x z$-plane and $x y$-plane form two other invariant families of planes, and we have; in all, three inviniant families of parallel planes, each family consisting of $\infty^{1}$ plawes.

The intersections of the plines parallel to the $y z$-plane and $x z$-plane are straight lines perpendicular to the $x y$-plane, and it is obvious, since the $\infty^{2}$ lines perpendicular to the $x y$-plane are the intersections of the planes of invariant families of planes, that these $\infty^{2}$ lines themselves form an invariant fumily of parallel lines. This, fumily of lines is invariant as a whole, while the lines of the family are interchanged among each other by means of the transformations of the $G_{\mathrm{n}}$.

Similarly the lines perpendicular to the yz-plane and $x z$-plane form two other invariant families of parallel lines, each family consisting of a liue congruence.

Since the planes of each invariant family of planes are parallel, the intersections of the parallel planes of each family will be a stritight line at infinity; and, as we have three invariant families of parallel planes, we shall have three such lines. It is clear, then, that these three straight lines at infinity are absolutely invariant.

4. To find what absolutely invariant loci exist within a finite distance of the origin, we equate to zero the coefficients of $p, q$, and $r$ in the general transformation; for they are the increments given to $x, y$, and $z$, respectively, by the infinitesimal transformation

$$
\nabla f=\left(a_{1}+2 b_{1} x+c_{1} x^{2}\right) p+\left(a_{2}+2 b_{2} y+c_{2} y^{2}\right) q+\left(a_{3}+2 b_{3} z+c_{3} z^{2}\right) r .
$$

Thus we have the equations

$$
\begin{aligned}
& a_{1}+2 b_{1} x+c_{1} x^{2}=0, \\
& a_{2}+2 b_{2} y+c_{2} y^{2}=0, \\
& a_{3}+3 b_{3} z+c_{3} z^{2}=0,
\end{aligned}
$$

which give the absolutely invariant planes

$$
x=\alpha_{1}, \quad x=\beta_{1}, \quad y=u_{2}, \quad y=\beta_{2}, \quad z=a_{3}, \quad z=\beta_{3},
$$


where the $\alpha$ 's and $\beta$ 's are the roots of the above equations, and have the values

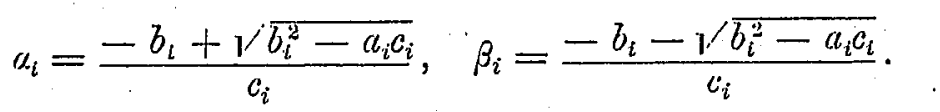

The intersections of these planes are, of course, invariant; hence the following points :

$$
\begin{array}{llll}
\left(\alpha_{1}, \alpha_{2}, \alpha_{3}\right), & \left(\alpha_{1}, \beta_{2}, \alpha_{3}\right), & \left(\beta_{1}, \alpha_{2}, \alpha_{3}\right), & \left(\beta_{1}, \beta_{2}, \alpha_{3}\right) \\
\left(\alpha_{1}, \alpha_{2}, \beta_{3}\right), & \left(\alpha_{1}, \beta_{2}, \beta_{3}\right), & \left(\beta_{1}, \alpha_{2}, \beta_{3}\right), & \left(\beta_{1}, \beta_{2}, \beta_{3}\right)
\end{array}
$$

are absolutely invariant points; and are the vertices of a parallelepipedon, which must also be absolutely invariant.

If $l_{i}{ }^{2}<a_{i} c_{i}$, the roots of the equations become imaginary, and our absolutely invariant planes, points, and parallelepipedon, likewise, become imaginary; for the imaginary planes do not intersect in any real points or point.

If $b_{i}^{2}=a_{i}{ }^{2}$, the roots of the equations are equal, and our planes now reduce to three and our parallelepipedon to an absolutely invariant point.

If $b_{i}=c_{i}=0, a_{1}=1, a_{2}=a_{3}=0$, the transformation becomes

$$
\sigma^{\prime} \equiv p \text {. }
$$

In this case, the roots of the equation

$$
a_{1}+2 b_{1} x+c_{1} x^{2}=0
$$

ase infinite, and the planes $x=$ const. are now at infiuity, while the planes . $y=$ const. and $z=$ const. are still invariant. A similar discussion holds when the transformation reduces to

$$
U f=q, \quad U f=r
$$

or to any mere translation.

5. The points in space are moved by the general transformation of the $G_{9}$ along $\infty^{2}$ curves, which we shall call the. Path-Curves of the Group. We shall now find these curves, and see whether any of the absolutely invariant points just found lie on this curve congruence.

These curves, as usual, are found by integrating, in the most general manner, the equations

$$
\frac{d x}{a_{1}+2 b_{1} x+c_{1} x^{2}}=\frac{d y}{a_{2}+2 b_{2} y+c_{2} y^{2}}=\frac{d z}{a_{3}+2 b_{3} z+c_{3} z^{2}} .
$$

We shall find them for the three principal cases only; that is, according as $a_{i} c_{i}<=>b_{i}^{2}(i=1,2,3)$. 
If $a_{i} c_{i}<b_{i}{ }^{2}$, the $\infty^{2}$ path-curves, as is readily seen, are given by the equations

where

$$
\begin{aligned}
& \frac{x-\alpha_{1}}{x-\beta_{1}}=C_{1}^{\prime}\left[\frac{y-\alpha_{2}}{y-\beta_{2}}\right]^{k}, \\
& \frac{x-\alpha_{1}}{x-\beta_{1}}=C_{2}\left[\frac{z-\alpha_{3}}{z-\beta_{3}}\right]^{h},
\end{aligned}
$$

$$
\begin{gathered}
\alpha_{i} \equiv \frac{-b_{i}+\sqrt{b_{i}^{2}-a_{i} c_{i}}}{c_{i}}, \quad \beta_{i} \equiv \frac{-b_{i}-\sqrt{b_{i}^{2}-a_{i} c_{i}}}{c_{i}}, \\
k^{2} \equiv \frac{b_{1}^{2}-a_{1} c_{1}}{b_{2}^{2}-a_{2} c_{2}}, \quad h^{2} \equiv \frac{b_{1}^{2}-a_{1} c_{1}}{b_{3}^{2}-a_{3} c_{3}},
\end{gathered}
$$

and $C_{1}$ and $C_{2}$ are constants of integration. Evidently the absolutely invariant points

$$
\begin{array}{ll}
P: & x=\alpha_{1}, \quad y=\alpha_{2}, \quad z=\alpha_{3}, \\
Q: & x=\beta_{1}, \quad y=\beta_{2}, \quad z=\beta_{3},
\end{array}
$$

lie on these curves, so that each curve of the congruence of path-curves passes through the points $P$ and $Q$.

If $a_{i} c_{i}>b_{i}{ }^{2}$, the $\infty^{2}$ path-curves are given by the equations

where

$$
\begin{aligned}
& \tan ^{-1}\left(\mu_{1} x+\nu_{1}\right)-\pi \tan ^{-1}\left(\mu_{2} y+\nu_{2}\right)=\text { const., } \\
& \tan ^{-1}\left(\mu_{1} x+\nu_{1}\right)-\pi \tan ^{-1}\left(\mu_{3} z+\nu_{3}\right)=\text { const. }
\end{aligned}
$$

$$
\mu_{t}=\frac{c_{i}}{\sqrt{a_{i} c_{t}-b_{i}^{2}}} \text { and } \nu_{i}-\frac{b_{i}}{\sqrt{a_{i} c_{i}-b_{i}^{2}}} \text {, }
$$

and $k$ and $h$ have the same values as above.

In this case, the absolutely invariant points become imaginary, since the imaginary planes do not intersect in any real points or point.

Finally, if $a_{i} c_{i}=b_{i}^{2}$, the $\infty^{2}$ path-curves are given by the equations

$$
\begin{aligned}
& \frac{1}{c_{1} x+b_{1}}-\frac{1}{c_{2} y+b_{2}}=\text { const. } \\
& \frac{1}{c_{1} x+b_{1}}-\frac{1}{c_{3} z+b_{3}}=\text { const. }
\end{aligned}
$$

or

$$
\begin{aligned}
& x y+m_{1} x+n_{1} y+b_{1}=0, \\
& x z+m_{2} x+n_{2} z+b_{2}=0 .
\end{aligned}
$$

In this case, the absolutely invariant points reduce to one point, the coordinates of which are

$$
x=-\frac{b_{1}}{c_{1}}, \quad y=-\frac{b_{2}}{c_{2}}, \quad z=-\frac{b_{3}}{c_{3}}
$$


This point lies on the curves just found, so that now each curve of the congruence of path-curves passes through one point.

In the first case the path-curves are usually algebraic; in the second, transcendental; and in the third, they are given as the intersections of hyperbotic cylinder's.

Of course, other cases may arise, but they need not be further discussed.

6. We have already found the points that are absolutely invariant under the transformations of the $G_{9}$. We shall now find what "Invariants" or" invariant relations exist between $n$ points, three points, and two points; that is, what functions $f\left(x_{1}, y_{1}, z_{1}, \ldots, x_{n}, y_{n}, z_{n}\right)$ are invariant, or what equations $f\left(x_{1}, y_{1}, z_{1}, \ldots, x_{n}, y_{n}, z_{n}\right)=0$, are invariant under the $G_{9}$.

We shall tirst define what is meant by an invariant function, and an invariant equation.

A function $f\left(x_{1}, y_{1}, z_{1}, \ldots, x_{n}, y_{n}, z_{n}\right)$ is said to be invariant uncler a group of transformations in the same variables, if each increment given to the function by each transformation of the group is identically zero.

An equation $f\left(x_{1}, y_{1}, z_{1}, \ldots, x_{n}, y_{n}, z_{n}\right)=0$ is said to be invariant, when the increments are either identically zero, or are zero by means of $f=0$. The analytical condition, then, is that the expression

$$
\partial f=\frac{\partial f}{\partial x_{1}} \delta x_{1}+\frac{\partial f}{\partial y_{1}} \delta y_{1}+\frac{\partial f}{\partial z_{1}} \delta z_{1}+\ldots+\frac{\partial f}{\partial z_{n}} \delta z_{n}
$$

shall be zero identically in case of an invariant function; and shall be zero identically or by means of $f=0$ in case of an invariant equation $f=0$,where the $\delta x_{i}, \delta y_{l}, \delta z_{i}$ must be obtained from the transformations of the Group.

7. We can now find the Invariants and invariant relations of $n$ points, three points, and two points.

Let the coordinates of the points be $x_{i}, y_{i}, z_{i}(i=1,2, \ldots, n)$. Here we wish, first, to find what functions $f\left(x_{1}, y_{1}, z_{1}, \ldots, x_{n}, y_{n}, z_{n}\right)$ are invariant.

In accordance with the above, we have the following conditions, which $f$ must satisfy simultaneously :

$$
\begin{aligned}
& U_{1} f=p_{1}+p_{2}+\ldots+p_{n}=0, \\
& U_{2} f=q_{1}+q_{2}+\ldots+q_{n}=0, \\
& U_{3} f=r_{1}+r_{2}+\ldots+r_{n}=0, \\
& U_{4} f=x_{1} p_{1}+x_{2} p_{2}+\ldots+x_{n} p_{n}=0, \\
& U_{5} f-y_{1} q_{1}+y_{2} q_{2}+\ldots+y_{n} q_{n}=0, \\
& U_{6} f=z_{1} r_{1}+z_{2} r_{2}+\ldots+z_{n} r_{n}=0 \\
& U_{7} f=x_{1}^{2} p_{1}+x_{2}^{2} p_{2}+\ldots+x_{n}^{2} p_{n}=0, \\
& U_{8} f=y_{1}^{2} q_{1}+y_{2}^{2} q_{2}+\ldots+y_{n}^{2} q_{n}=0, \\
& U_{9} f \equiv z_{1}^{2} r_{1}-z_{2}^{2} r_{2}+\ldots+z_{n}^{2} r_{n}=0 .
\end{aligned}
$$


ROBERTS. ON THE TRANSFORMATION GROUP, ETC.

The above complete system consists of nine equations in $3 n$. variables; and, since all the n-row determinants of the matrix of the coefficients of $p_{i}, q_{i}, r_{i}$ do not become zero identically, there are $3(n-3)$ solutions of the system. From equations $U_{1} f, U_{4} f$, and $U_{7} f$ we find $(n-3)$ functions $f\left(x_{1}, x_{2}, \ldots, x_{n}\right)$, which are solutions of the system, as none of the other equations contain $x_{i}$. In order to find these $(n-3)$ solutions, we proceed as follows (see $\$ 13)$ :

'The solutions of equation $U_{\text {s }} f$ are

$$
\frac{x_{2}}{x_{1}} \equiv \alpha_{1}, \quad \frac{x_{3}}{x_{1}} \equiv \alpha_{n 2}, \quad \ldots, \quad \frac{x_{n}}{x_{1}} \equiv a_{n-1} \text {. }
$$

If we introduce the quantities $\alpha_{i}$, as new variables, into the equations $U_{1} f$ and $U_{2} f$, - since

$$
\begin{gathered}
U_{1} \alpha_{1}=\frac{1}{x_{1}}\left(1-\alpha_{1}\right), \quad U_{1} \alpha_{2}=\frac{1}{x_{1}}\left(1-\alpha_{2}\right), \ldots, \quad U_{1} \alpha_{n-1}=\frac{1}{x_{1}}\left(1-\alpha_{n-1}\right), \\
U_{7} \alpha_{1}=-x_{1} \alpha_{1}\left(1-\alpha_{1}\right), U_{7} \alpha_{n_{2}}=-x_{1} \alpha_{2}\left(1-\alpha_{2}\right), \ldots, U_{7} \alpha_{n-1}=-x_{1} \alpha_{n-1}\left(1-\alpha_{n-1}\right),
\end{gathered}
$$

we have the following equations:

$$
\begin{gathered}
U_{10} f=\left(1-\alpha_{1}\right) \frac{\partial f}{\partial \alpha_{1}}+\left(1-u_{2}\right) \frac{\partial f}{\partial \alpha_{2}}+\ldots+\left(1-u_{n-1}\right) \frac{\partial f}{\partial \alpha_{n-1}}=0 \\
U_{11} f=\alpha_{1}\left(1-\alpha_{1}\right) \frac{\partial f}{\partial u_{1}}+\alpha_{-2}\left(1-u_{2}\right) \frac{\partial f}{\partial \alpha_{2}}+\ldots+\alpha_{n-1}\left(1-\alpha_{n-1}\right) \frac{\partial f}{\partial u_{n-1}}=0 .
\end{gathered}
$$

The solutions of equation $U_{10} f$ are

$$
\frac{1-\alpha_{12}}{1-\alpha_{1}}=\beta_{1}, \ldots, \frac{1-\alpha_{n-1}}{1-\alpha_{1}}=\beta_{n-2} .
$$

If we introduce the quantities $\beta_{i}$, as new variables, into the equation $U_{11} f,-\operatorname{since}$

$$
U_{11} \beta_{1}=\left(1-a_{1}\right) \beta_{1}\left(\beta_{1}-1\right), \ldots, \quad U_{11} \beta_{n-2}=\left(1-\alpha_{1}\right) \beta_{n-2}\left(\beta_{n-2}-1\right),
$$

we have the equation

$$
U_{12} f \equiv \beta_{1}\left(\beta_{1}-1\right) \frac{\partial f}{\partial \beta_{1}}+\ldots+\beta_{n-2}\left(\beta_{n-2}-1\right) \frac{\partial f}{\partial \beta_{n-2}}=0
$$

The solutions of this equation are

$$
\begin{aligned}
& \frac{\beta_{1}-1}{\beta_{1}} \cdot \frac{\beta_{2}}{\beta_{2}-1}=\frac{x_{2}-x_{3}}{x_{1}-x_{2}} \cdot \frac{x_{1}-x_{4}}{x_{2}-x_{4}}, \\
& \cdot \cdot \cdot \cdot \cdot \cdot \cdot \cdot \cdot \cdot \cdot \cdot \cdot \cdot . \\
& \frac{\beta_{1}-1}{\beta_{1}} \cdot \frac{\beta_{n-2}}{\beta_{n-2}-1}=\frac{x_{2}-x_{3}}{x_{1}-x_{2}} \cdot \frac{x_{1}-x_{n}}{x_{2}-x_{n}} .
\end{aligned}
$$


We can now, on account of the symmetry of the equations, write down the remaining $2(n-3)$ solutions. They are

where $(i=4,5, \ldots, n)$.

$$
\begin{gathered}
f \equiv \frac{y_{2}-y_{3}}{y_{1}-y_{2}} \cdot \frac{y_{1}-y_{i}}{y_{2}-y_{i}} \\
f \equiv \frac{z_{2}-z_{3}}{z_{1}-z_{2}} \cdot \frac{z_{1}-z_{i}}{z_{2}-z_{i}}
\end{gathered}
$$

Hence, if four points, whose $x$ 's, $y$ 's, or $z$ 's form an anharmonic ratio, are transformed by means of the transformations of the Group, the $x$ 's, $y$ 's, or $z$ 's of the new positions of the four points will form the same anharmonic ratio.

Evidently, this is equivalent to saying that the anharmonic ratio of four planes parallel to one of the coordinate planes is invariant.

For the invariant relations of two points, we lave to find what equations $f\left(x_{i}, y_{i}, z_{i}, x_{j}, y_{j}, z_{j}\right)=0$ satisfy all the partial differential equations, either identically or by means of $f^{\prime}=0$.

It is readily seen that the equations

$$
f \equiv x_{i}-x_{j}=0, f \equiv y_{i}-y_{j}=0, f \equiv z_{i}-z_{j}=0
$$

satisfy all the partial differential equations by menns of $f=0$; hence $f=0$, in any of the above forms, is an invariant equation. This means, - when, for example, $x_{1}-x_{2}=0$ is the invariant relation of two points, -that, if the two points lie in a plane parallel to the yz-plane, when they are transformed by the transformations of the Group they will still lie in a plane parallel to the yz-plane.

It may be shown that all the invariant relations of two points are of the above forms.

Similarly, for three points, we have the equations

$$
\begin{aligned}
& x_{i}-x_{j}=0, \quad x_{i}-x_{k}=0, \quad x_{j}-x_{i}=0, \\
& y_{i}-y_{j}=0, \quad y_{i}-y_{k}=0, \quad y_{j}-y_{i}=0, \\
& z_{i}-z_{j}=0, \quad z_{i}-z_{k}=0, \quad z_{j}-z_{k}=0 .
\end{aligned}
$$

These results are interpreted just as in the case of two points.

8. In like manner we can find the Invariants and inviniant relations of a point and a plane, two points and a plane, a plane and two points, etc.

Writing the equation to the plane in the form

$$
u x+v y+w z=1
$$


and writing the transformations of the Group in the variables $u$, $v$, and $w$, the problem becomes to find what functions

$$
\begin{gathered}
f_{1}\left(x_{1}, y_{1}, z_{1}, u, v, w\right), f_{2}\left(x_{1}, y_{1}, z_{1}, x_{2}, y_{2}, z_{2}, u, v, w\right), \\
f_{3}\left(x_{1}, y_{1}, z_{1}, u_{1}, v_{1}, w_{1}, u_{2}, v_{2}, w_{2}\right), \text { etc. }
\end{gathered}
$$

and what equations

$$
f_{1}=0, f_{2}=0, f_{3}=0, \text { etc., }
$$

are invariant under the Group.

The invariant functions, and invariant equations, are found in a manner entirely analogous to that of the preceding paragraph; and it will not be necessary to go through with the work here.

9. It will be interesting to know what will happen, if we make some point absolutely invariant; that is, hold some point of general position as fixed.

'The plane passing through the fixed point parallel to one of the coordivate planes is invariant, since the transformations of the Gronp move all such planes parallel to themselves $(\S 3)$. Two of the invariant planes of $\S 4$ are parallel to it. Suppose these three planes are given by the equations.

$$
x=\gamma_{1}, \quad x=\alpha_{1}, \quad x=\beta_{1} .
$$

The plane which forms an anharmonic ratio with these three planes is absolutely invariant ( $\$ 7$ ). Let this plane be given by the equation

$$
x=\varepsilon_{1} \text {. }
$$

The plane which forms an anharmonic ratio with the planes

$$
x=\varepsilon_{1}, \quad x=\gamma_{1}, \quad x=\alpha_{1},
$$

is also absolutely invariant; thus there is found another absolutely invariant plane; and by this method there can be found $\infty^{1}$ absolutely invariant planes parallel to the $y z-$ plane; $\infty{ }^{1}$, parallel to the $x z=-p l a n e$; and $\infty^{1}$, parallel to the $x y$-plane. These three families of absolutely invariant planes intersect in $\infty^{3}$ points, which, of course, are absolutely invariant. Hence every point in space becomes absolutely invariant, if a point of general position is held fixed.

10. The results of the last paragraph may be shown analytically.

Let the coordinates of the fixed point, which does not lie on any of the absolutely invariant planes, be

$$
x=r_{1}, \quad y=r_{2}, \quad z=r_{3} .
$$

These values of $x, y$, and $z$, by hypothesis, reduce the transformation

$$
U f \equiv\left(a_{1}+2 b_{1} x+c_{1} x^{2}\right) p+\left(a_{2}+2 b_{2} y+c_{2} y^{2}\right) q+\left(a_{3}+2 b_{3} z+c_{3} z^{2}\right) r
$$


to the identical transformation ; therefore, these values must satisfy the equations

$$
\begin{aligned}
& a_{1}+2 b_{1} x+c_{1} x^{2}=0, \\
& a_{2}+2 b_{2} y+c_{2} y^{2}=0, \\
& a_{3}+2 b_{3} z+c_{3} z^{2}=0 .
\end{aligned}
$$

We, consequently, have the three following conditions, from which $a_{i}, b_{i}$, and $c_{i}$ may be determined

$$
\begin{aligned}
& a_{i}+2 b_{i i_{i}}+c_{i} \gamma_{i}^{2}=0, \\
& a_{i}+2 b_{i} \mu_{i}+c_{i} \mu_{i}^{2}=0, \\
& a_{i}+2 b_{i} \beta_{i}+c_{i} \beta_{i}^{2}=0,
\end{aligned}
$$

where $\alpha_{i}$ and $\beta_{i}$ are the roots of the equations (\$4).

Eliminating $a_{i}$, we have

$$
\begin{aligned}
& c_{i}\left(\alpha_{i}+\gamma_{i}\right)+2 b_{i}=0, \\
& c_{i}\left(\alpha_{i}+\beta_{i}\right)+2 b_{i}=0 .
\end{aligned}
$$

Eliminating $\delta_{i}$, wo have

$$
c_{i}\left(\gamma_{i}-\beta_{i}\right)=0 \text {, }
$$

but since $\gamma_{i}$ cannot equal $\beta_{i}$, we must have $c_{i}=0$, and, consequently, $a_{i}=b_{t}$ $=0$. Now; if $a_{i}=b_{i}=c_{i}=0$, the coefficients of $p, q$, and $r$ are zero. Since these coefficients are the increments given to $x, y$, aud $\approx$, respectively, by means of the transformation, and since they are zero, the trunsformation reduces to the identical transformation.

11. The invariant families, so far found, have been either planes or straight lines. We shall now show that the Group may be put into such a form that it will leave the families of all surfuces of rotation

invariant.

$$
z=\varphi\left(i c^{2}+y^{2}+a x+b y+c\right)
$$

We only need show, to this end, that the Sub-Group

$$
p, \quad q, \quad x p, \quad y q, \quad x^{2} p, \quad y^{2} q
$$

can be put into such form that it leaves the $\infty^{3}$ circles in the $x y$-plane invariant as a family; for the transformation $z r$ and $z^{2} r$ do not transform this plane at all, while the transformation $r$ merely translates it. 
Introduce into the Sub-Group the functions $x_{1}$ and $y_{1}$, as new variables, by means of

$$
\begin{aligned}
& x=x_{1}+i y_{1}, \\
& y=x_{1}-i y_{1} ;
\end{aligned}
$$

then $p \equiv \frac{\partial f}{\partial x}$ and $q \equiv \frac{\partial f}{\partial y}$ become, in these variables,

and

$$
\frac{\partial f}{\partial x_{1}}+\frac{1}{i} \frac{\partial f}{\partial y_{1}}
$$

$$
\frac{\partial f}{\partial x_{1}}-\frac{1}{i} \frac{\partial f}{\partial y_{1}}
$$

or $i p_{1}+q_{1}$ and $i p_{1}-q_{1}$; and the transformations, as is readily seen, may be written in the forms

$$
\begin{gathered}
i p_{1}+q_{1}, \quad i p_{1}-q_{1} \\
x_{1} q-y_{1} p_{1}+i\left(x_{1} p_{1}+y_{1} q_{1}\right), \quad x_{1} q_{1}-y_{1} p_{1}-i\left(x_{1} p_{1}+y_{1} q_{1}\right) \\
\left(x_{1}^{2}-y_{1}^{2}\right) q_{1}-2 x_{1} y_{1} p_{1}+i\left\{\left(x_{1}^{2}-y_{1}^{2}\right) p_{1}+2 x_{1} y_{1} q_{1}\right\} \\
\left(2 x_{1} y_{1} p_{1}-\left(x_{1}^{2}-y_{1}^{2}\right) q_{1}+i\left\{\left(x_{1}^{2}-y_{1}^{2}\right) p_{1}+2 x_{1} y_{1} q_{1}\right\}\right.
\end{gathered}
$$

Hence the $G_{6}$ may be put in the form

$$
p, \quad q, \quad y p-x q, \quad x p+y q, \quad\left(x^{2}-y^{2}\right) p+2 x y q, \quad 2 x y p-\left(x^{2}-y^{2}\right) q
$$

The finite transformations of this $G_{6}$ are (\$2)

$$
\begin{aligned}
& x_{1}=\frac{\alpha_{1}(x+i y)+\alpha_{2}}{2\left(\alpha_{3}(x+i y)+1\right)}+\frac{\beta_{1}(x-i y)+\beta_{2}}{2\left(\beta_{3}(x-i y)+1\right)}, \\
& y_{1}=\frac{1}{2 i}\left[\frac{\alpha_{1}(x+i y)+\alpha_{2}}{\alpha_{3}(x+i y)+1}-\frac{\beta_{1}(x-i y)+\beta_{2}}{\beta_{3}(x-i y)+1}\right] .
\end{aligned}
$$

Let the equation to the circles in the $x y$-plane be

$$
\Phi \equiv x_{1}^{2}+y_{1}^{2}+a x_{1}+b y_{1}+c=0 \text {. }
$$

Substituting in this equation the above values of $x_{1}$ and $y_{1}$, we get

$$
\begin{gathered}
\frac{\left\{\alpha_{1}(x+i y)+\alpha_{2}\right\}\left\{\beta_{1}(x-i y)+\beta_{2}\right\}}{\left\{\alpha_{3}(x+i y)+1\right\}\left\{\beta_{3}(x-i y)+1\right\}}+\frac{a}{2}\left\{\frac{\alpha_{1}(x+i y)+\alpha_{2}}{\alpha_{3}(x+i y)+1}+\frac{\beta_{1}(x-i y)+\beta_{2}}{\beta_{3}(x-i y)+1}\right\} \\
+\frac{b}{2 i}\left\{\frac{\alpha_{1}(x+i y)+\alpha_{2}}{\alpha_{3}(x+i y)+1}-\frac{\beta_{1}(x-i y)+\beta_{2}}{\beta_{3}(x-i y)+1}\right\}+c=0,
\end{gathered}
$$


ROBERTS. ON THE TRANSFORMATION GROUP, ETO.

which may be reduced to

$$
x^{2}+y^{2}+G x+H y+F=0 .
$$

Hence the circles $\phi=0$ are invariant, as a family.

In the form

$$
\begin{gathered}
p, q, \quad y p-x q, \quad x p+y q \\
\left(x^{2}-y^{2}\right) p+2 x y q, \quad 2 x y p-\left(x^{2}-y^{2}\right) q \\
r, \quad z r, \quad z^{2} r
\end{gathered}
$$

the $G_{9}$ always transforms a circle in the $x y$-plane into a circle, since $z r$ and $z^{2} r$ do not transform that plane at all, while $r$ translates it merely. Hence a surface which cuts that plane in a circle must be transformed into a surface which also cuts it in a circle, or the surfaces of rotation

$$
z=\varphi\left(x^{2}+y^{2}+a x+b y+c\right)
$$

are an invarnant family under the Group as written above.

This is readily verified from the form of the equation of surfaces of rotation

$$
z=\varphi\left(x^{2}+y^{2}+a x+b y+c\right) \text {. }
$$

II.

\section{Differential Intariants.}

Intariant Differential EQUations.

12. In this section we shall assume $y$ and $z$ to he functions of $x$; and shall proceed to find the Differential Invariants of the lowest order of the $G_{9}$, and then show how the Differential Invariants of higher orders may be found.

We shall also find what ordinary differential equations are invariant under the $G_{9}$.

13. We shall make frequent use of the following theorem from the theory of the complete system :

If $A_{1} f=0, \ldots, A_{r} f=0$ form a complete system in $x_{1}, \ldots, x_{n}(r<n)$, the solutions of the same can be obtained in the following manner. We find the solutions $\varphi_{1}, \ldots, \varphi_{n-1}$ of $A_{1} f=0$; and then write $A_{2} f=0$ in the form

$$
A_{2} f \equiv A_{2} \varphi_{1} \frac{\partial f}{\partial \varphi_{1}}+\ldots+A_{2} \varphi_{n-1} \frac{\partial f}{\partial \varphi_{n-1}}=0
$$


If the ratios of the $A_{2} \varphi_{k}$ ar'c not functions of $\varphi_{1}, \ldots, \varphi_{n-1}$ alone, the equation $A_{2} f=0$ breates up into several equations. We integrate one of these and introduce the corresponding solutions $\phi_{1}, \ldots, \phi_{n-2}$ into $A_{3} f=0$. The resulting equation

$$
A_{3} \psi_{1} \frac{\partial f}{\partial \psi_{1}}+\ldots=0
$$

we handle in an anclogous manner, etc. Thus we find ultimately the $(n-r)$ solutions of the complete system.

14. To Find the Differential Invariant of the Lowest Order.

Here we have to find what functions $f\left(x, y, z, y_{1}, z_{1}, \ldots, y_{n}, z_{n}\right)$ are invariant under the transformations of the Group,-where

Now

$$
y_{i} \equiv \frac{d^{i} y}{d x^{i}}, \quad z_{i} \equiv \frac{d^{i} \xi}{d x^{i}}
$$

$$
\delta f=\frac{\partial f}{\partial x} \partial x+\frac{\partial f}{\partial y} \partial y+\frac{\partial f}{\partial y_{1}} \partial y_{1}+\ldots+\frac{\partial f}{\partial z_{n}} \partial z_{n}
$$

and we must obtain the increments $\delta y_{i}, \partial z_{l}$ for each trausformation of the Group.

If we have given any transformation

$$
U f \equiv \xi \frac{\partial f}{\partial x}+\eta \frac{\partial f}{\partial y}+\zeta \frac{\partial f}{\partial z}
$$

we find $\delta y_{1}, \delta z_{1}, \ldots, \delta y_{n}, \delta z_{n}$ as follows :

so that

$$
y_{1} d x=d y
$$

or

$$
\begin{aligned}
& \delta y_{1} d x+y_{1} \partial d x=\delta d y, \\
& \partial y_{1} d x+y_{1} d \partial x=d \delta y, \\
& \therefore \delta y_{1}=\frac{d \partial y}{d x}-y_{1} \frac{d \partial x}{d x}
\end{aligned}
$$

Since $\delta x=\xi \delta t$ and $\delta y=\eta \partial t$, we have

$$
\frac{\partial y_{1}}{\partial t}=\frac{d \eta}{d x}-y_{1} \frac{d \xi}{d x} \equiv \eta_{1} \text { (say). }
$$

Similarly,

$$
\frac{\partial z_{1}}{\partial t}=\frac{d \zeta}{d x}-z_{1} \frac{d \xi}{d x} \equiv \zeta_{1} \text { (say) }
$$


ROBERIS. ON THE TRANSFORMATION GROUP, ETC.

In an analogous manner, we find

$$
\begin{aligned}
& \eta_{i}=\frac{d \eta_{l-1}}{d x}-y_{i} \frac{d \xi}{d x} \\
& \zeta_{i}=\frac{d \zeta_{i-1}}{d x}-z_{i} \frac{d \xi}{d x} .
\end{aligned}
$$

We may now write the transformation in the form

$$
U_{n} f \equiv \xi \frac{\partial f}{\partial x}+\eta \frac{\partial f}{\partial y}+\zeta \frac{\partial f}{\partial z}+\ldots+\eta_{n} \frac{\partial f}{\partial y_{n}}+\zeta_{n} \frac{\partial f}{\partial z_{n}}
$$

and we then say that the transformation has been extended $n$ times.

We obtain a complete system of nine equations in nine variables, if we find the increments $\delta y_{i}$ and $\delta z_{i}(i=1,2,3)$ for each transformation of the Group, substitute them in equation (1), and put $i_{j} f=0(j=1,2, \ldots, 9)$; or, what amounts to the same thing, if we extend each transformation three times, and then put each extended transformation equal to zero.

Extending each transformation of the $G_{9}$ three times, and putting ench extended trausformation equal to zero, we obtain the following complete system,-in which $q_{i} \equiv \frac{\partial f}{\partial y_{i}}$, etc.

$$
\begin{aligned}
& \quad p=0, \quad q=0, \quad r=0, \\
& x p-y_{1} q_{1}-z_{1} r_{1}-2 y_{2} q_{2}-2 z_{2} r_{2}-3 y_{3} q_{3}-3 z_{3} r_{3}=0, \\
& y q+y_{1} q_{1}+y_{2} q_{2}+y_{3} q_{3}=0, \\
& z r+z_{1} r_{1}+z_{2} r_{2}+z_{3} r_{3}=0, . \\
& x^{2} p-2 x y_{1} q_{1}-2 x z_{1} r_{1}-2\left(y_{1}+2 x y_{2}\right) q_{2}-2\left(z_{1}+2 x z_{2}\right) r_{2}-6\left(y_{2}+x y_{3}\right) q_{3} \\
& \quad-6\left(z_{2}+x z_{3}\right) r_{3}=0, \\
& y^{2} q+2 y y_{1} q_{1}+2\left(y_{1}^{2}+y y_{2}\right) q_{2}+2\left(3 y_{1} y_{2}+y y_{3}\right) q_{3}=0, \\
& z^{2} r+2 z z_{1} r_{1}+2\left(z_{1}^{2}+z z_{2}\right) r_{2}+2\left(3 z_{1} z_{2}+z z_{3}\right) r_{3}=0 .
\end{aligned}
$$

From the first three equations, we see that the solutions, if any exist, will be free of $x, y$, and $z$; hence, we may neglect those terms containing $p, q$, and $r$. Doing this, and reducing algebraically, the system becomes

$$
\begin{aligned}
& U_{1} f \equiv y_{1} q_{1}+z_{1} r_{1}+2 y_{2} q_{2}+2 z_{2} r_{2}+3 y_{3} q_{3}+3 z_{3} r_{3}=0 \\
& U_{2} f \equiv y_{1} q_{1}+y_{2} q_{2}+y_{3} q_{3}=0 \\
& U_{3} f \equiv z_{1} r_{1}+z_{2} r_{2}+z_{3} r_{3}=0 \\
& U_{4} f \equiv y_{1} q_{2}+z_{1} r_{2}+3 y_{2} q_{3}+3 z_{2} r_{3}=0 \\
& U_{5} f \equiv y_{1} q_{2}+3 y_{2} q_{3}=0 \\
& U_{6} f \equiv z_{1} r_{2}+3 z_{2} r_{3}=0
\end{aligned}
$$


Since $U_{4} f$ is the sum of $\dot{U}_{5} f$ and $U_{f_{e}} f$, the system reduces to one of five equations in six variables; hence there exists at least one solution.

The matrix of the coefficients of $q_{i}$ and $r_{i}$ is

$$
\left\|\begin{array}{llllll}
y_{1} & z_{1} & 2 y_{2} & 2 z_{2} & 3 y_{3} & 3 z_{3} \\
y_{1} & 0 & y_{2} & 0 & y_{3} & 0 \\
0 & z_{1} & 0 & z_{2} & 0 & z_{3} \\
0 & 0 & y_{1} & 0 & 3 y_{2} & 0 \\
0 & 0 & 0 & z_{1} & 0 & 3 z_{2}
\end{array}\right\| .
$$

All the determinants of this matrix do not vanish identically; hence there is one and only one solution of the complete system.

In order to find the solution we proceed as follows: (Cf. $\$ 13$ )

The solutions common to $U_{5} f$ and $U_{6} f$ are

$$
\frac{y_{3}}{y_{1}}-\frac{3}{2}\left[\frac{y_{2}}{y_{1}}\right]^{2} \equiv a, \frac{z_{3}}{z_{1}}-\frac{3}{2}\left[\frac{z_{2}}{z_{1}}\right] \equiv \beta
$$

which are also solutions of $U_{2} f$ and $U_{3} f$.

Introducing these solutions, as new variables, in $U_{1} f,-\operatorname{since}$

we have the equation

$$
U_{1} \alpha=2 \kappa, \quad U_{1} \beta=2 \beta,
$$

$$
U_{7} f \equiv \alpha \frac{\partial f}{\partial \alpha}+\beta \frac{\partial f}{\partial \beta}=0 \text {, }
$$

the solution of which is

$$
\frac{\beta}{\alpha} \equiv \frac{y_{1}^{2}\left(2 z_{1} z_{3}-3 z_{2}^{2}\right)}{z_{1}^{2}\left(2 y_{1} y_{3}-3 y_{2}^{2}\right)}
$$

Thus we have found the solution of the complete system : which is the Differential Invariant of the $G_{9}$ of the third order.

15. To Find the Differential Invariants of Higher Orders.*

Suppose $\Omega\left(x, y, z, y_{1}, z_{1}, \ldots, \varphi, \varphi_{1}, \varphi_{2}, \ldots\right)$ to be such a function that, when $\varphi$ is any Differential Invariant of the Group, $\Omega$ is also a Differential Invariant, - to find the function $\Omega$.

We have

so that

$$
\varphi_{1} d x=d \varphi
$$

$$
\hat{o} \varphi_{1} d x+\varphi_{1} d \hat{\partial} x=d \hat{\delta} \varphi
$$


or

$$
\delta \varphi_{1}=\frac{d \dot{\delta} \varphi}{d x}-\varphi_{1} \frac{d \delta x}{d x}
$$

but, $\operatorname{sinc} \theta \varphi$ is an invariant, $\delta \varphi=0$, and we have

$$
\delta \varphi_{1}=-\varphi_{1} \frac{d \delta x}{d x}
$$

Now:

$$
\delta \Omega=\frac{\partial \Omega}{\partial x} \delta x+\ldots+\frac{\partial \Omega}{\partial y_{1}} \delta y_{1}+\ldots+\frac{\partial \Omega}{\partial \varphi} \delta \varphi+\frac{\partial \Omega}{\partial \varphi_{1}} \delta \varphi_{1}
$$

and since $\delta \Omega$ must be zero for every transformation of the Group, we have the following complete system, from which $\Omega$ may be determined :

$$
\begin{aligned}
& \quad p=0, \quad q=0, \quad r=0, \\
& x p-y_{1} q_{1}-z_{1} r_{1}-2 y_{2} q_{2}-2 z_{2} r_{2}-3 y_{3} q_{3}-\varphi_{1} \frac{\partial \Omega}{\partial \varphi_{1}}=0, \\
& y q+y_{1} q_{1}+y_{2} q_{2}+y_{3} q_{3}=0, \\
& z r+z_{1} r_{1}+z_{2} r_{2}=0, \\
& x^{2} p-2 x y_{1} q_{1}-2 x z_{1} r_{1}-2\left(y_{1}+2 x y_{2}\right) g_{2}-2\left(z_{1}+2 x z_{2}\right) r_{2}-6\left(y_{2}+x y_{3}\right) q_{3} \\
& \cdot \\
& y^{2} q+2 y y_{1} q_{1}+2\left(y_{1}^{2}+y y_{2}\right) q_{2}+2\left(3 y_{1} y_{2}+y y_{3}\right) q_{3}=0, \\
& z^{2} r+2 z z_{1} r_{1}+2\left(z_{1}^{2}+z z_{2}\right) r_{2}=0,
\end{aligned}
$$

where

$$
p \equiv \frac{\partial Q}{\partial x}, \quad q_{i} \equiv \frac{\partial \mathscr{Q}}{\partial y_{i}}, \quad r_{i} \equiv \frac{\hat{c} \mathscr{Q}}{\partial z_{i}} .
$$

The first three equations show that the solutions will be independent of $x, y$, and $z$; hence, we neglect those terms containing $p, q$, and $r$.

By algebraic reduction these equations become

$$
\begin{aligned}
& U_{1} \Omega \equiv y_{1} q_{1}+z_{1} r_{1}+2 y_{2} q_{2}+2 z_{2} r_{2}+3 y_{3} q_{3}+\varphi_{1} \frac{\partial Q_{1}}{\partial \varphi_{1}}=0 \\
& U_{2} \Omega \equiv y_{1} q_{1}+y_{2} q_{2}+y_{3} q_{3}=0 \\
& U_{3} \Omega \equiv z_{1} r_{1}+z_{2} r_{2}=0 \\
& U_{4} \Omega \equiv y_{1} q_{2}+z_{1} r_{2}+3 y_{2} q_{3}=0 \\
& U_{5} \Omega \equiv y_{1} q_{2}+3 y_{2} q_{3}=0 \\
& U_{6} \Omega=z_{1} r_{2}=0
\end{aligned}
$$


We see from $U_{3} Q$ and $\sigma_{6} Q$ that the solution will be free of $z_{1}, z_{2}$, and $z_{3}$. The solutions common to $U_{2} \Omega$ and $U_{5} \Omega$ are

$$
\frac{y_{3}}{y_{1}}-\frac{3}{2}\left[\frac{y_{2}}{y_{1}}\right]^{2} \equiv \psi, \text { and } \varphi_{1} \text {. }
$$

Introduce these, as new variables, into $U_{1} \Omega$; hence, as

we have the equation

$$
U_{1} \alpha=2 \%, \quad U_{1} \varphi_{1}=\varphi_{1},
$$

$$
U_{7} \Omega \equiv 2 \alpha \frac{\partial \Omega}{\partial \alpha}+\varphi_{1} \frac{\partial \Omega}{\partial \varphi_{1}}=0
$$

The solution of this equation is

$$
\Delta \varphi \equiv \frac{\varphi_{1}}{u^{1 / 2}}
$$

$\Delta \varphi$ is called a "Differential Parameter" and has the general form

$$
\Omega\left(x, y, z, y_{1}, z_{1}, \ldots, \varphi, \Delta \varphi\right)
$$

Since $d \varphi$ is an Invariant,

$$
\Delta^{2} \varphi \equiv \Delta(\Delta \varphi) \equiv \frac{d \Delta \varphi}{\frac{d x}{a^{1 / t}}}
$$

is also a Differential Parameter, and likewise $\Delta^{3} \varphi$, etc., hence the most general Differential Parameter is

$$
Q\left(x, y, z, y_{1}, z_{1}, \ldots, \varphi, \Delta \varphi_{1}, \Delta^{2} \varphi, \ldots\right) .
$$

16. We shall now make use of the Differential Parameter to find one of the Differential Invariants of the fourth order.

Let

$$
\varphi \equiv \frac{\beta}{\alpha}
$$

where

Now

$$
\beta \equiv \frac{z_{3}}{z_{1}}-\frac{3}{2}\left[\frac{z_{2}}{z_{1}}\right]^{2}, \alpha \equiv \frac{y_{3}}{y_{1}}-\frac{3}{2}\left[\frac{y_{2}}{y_{1}}\right]^{2} .
$$

$$
\varphi_{1}=\frac{d \varphi}{d x}=\frac{\beta_{1} \alpha-\alpha_{1} \beta}{\alpha^{2}}
$$

where

$$
\beta_{1} \equiv \frac{d \beta}{d x}, \quad \alpha_{1} \equiv \frac{d \alpha}{d x}
$$

Hence

$$
\Delta \varphi \equiv \frac{\varphi_{1}}{\alpha^{1 / 2}} \equiv \frac{\beta_{1} \alpha-\alpha_{1} \beta}{\alpha^{5 / 2}}
$$

is a Differential Invariant of the fourth order. 
ROBERTS. ON THE TRANSFORMLIION GROUP, ETC.

17. To Find the Other Differential Invariants of the Fourth Order.

By extending each transformation of the $G_{9}$ four times, and by reducing the equation algebraically, remembering that the solutions will be free of $x, y$, and $z$, we get following complete system :

$$
\begin{aligned}
& U_{1} f \equiv y_{1} q_{1}+z_{1} r_{1}+2 y_{2} q_{2}+2 z_{3} r_{2}+3 y_{3} q_{3}+3 z_{3} r_{3}+4 y_{4} q_{4}+4 z_{4} r_{4}=0, \\
& U_{2} f \equiv y_{1} y_{1}+y_{2} q_{2}+y_{3} q_{3}+y_{4} q_{4}=0 \\
& U_{3} f \equiv z_{1} r_{1}+z_{2} r_{2}+z_{3} r_{3}+z_{4} r_{4}=0 \\
& U_{4} f \equiv y_{1} q_{2}+z_{1} r_{2}+3 y_{2} q_{3}+3 z_{2} r_{3}+6 y_{3} z_{4}+6 z_{3} r_{4}=0 \\
& U_{3} f \equiv y_{1}^{2} q_{2}+3 y_{1} y_{2} y_{3}+\left(3 y_{2}^{2}+4 y_{1} y_{3}\right) q_{4}=0 \\
& U_{6} f \equiv z_{1}^{2} r_{2}+3 z_{1} z_{2} r_{3}+\left(3 z_{2}^{2}+4 z_{1} z_{3}\right) r_{4}=0 .
\end{aligned}
$$

This is a complete system of six equations in eight variables, aud has at least two solations.

The matrix of the coefficients of $q_{l}, r_{l}$ is

$$
\left\|\begin{array}{cccccccc}
y_{1} & z_{1} & 2 y_{2} & 2 z_{2} & 3 y_{3} & 3 z_{3} & 4 y_{1} & 4 z_{1} \\
y_{1} & 0 & y_{2} & 0 & y_{3} & 0 & y_{4} & 0 \\
0 & z_{1} & 0 & z_{2} & 0 & z_{3} & 0 & z_{4} \\
0 & 0 & y_{1} & z_{1} & 3 y_{2} & 3 z_{2} & 6 y_{3} & 6 z_{3} \\
0 & 0 & y_{1}^{2} & 0 & 3 y_{1} y_{2} & 0 & \left(3 y_{2}^{2}+4 y_{1} y_{3}\right) & 0 \\
0 & 0 & 0 & z_{1}^{2} & 0 & 3 z_{1} z_{2} & 0 & \left(3 z_{2}^{2}+4 z_{1} z_{3}\right)
\end{array}\right\| .
$$

All the determinants of this matrix do not vanish identically; hence there are only two independent solutions of the complete system.

In order to find the solutions of the complete system, we proceed as follows (see $\$ 13$ ):

The solutions common to $U_{2} f$ and $U_{3} f$ are

$$
\begin{aligned}
& \frac{y_{2}}{y_{1}}: a_{2}, \frac{y_{3}}{y_{1}}=a_{3}, \frac{y_{4}}{y_{1}}=a_{4}, \\
& \frac{z_{2}}{z_{1}} \equiv b_{2}, \frac{z_{3}}{z_{1}} \equiv b_{3}, \frac{z_{4}}{z_{1}} \ldots b_{4} .
\end{aligned}
$$

Introduce these solutions, as new variables, into $U_{5 i} f$ and $U_{6} f$,-since

$$
\begin{array}{lll}
U_{5} a_{2}=y_{1}, & U_{5} a_{3}=3 a_{2} y_{1}, . & U_{5} a_{4}=\left(3 a_{2}^{2}+4 a_{3}\right) y_{1}, \\
U_{6} b_{2}=z_{1}, & U_{6} b_{3}=3 b_{2} z_{1}, & U_{6} b_{4}=\left(3 b_{2}^{2}+4 b_{3}\right) z_{1},
\end{array}
$$


we have the equations

$$
\begin{aligned}
& U_{7} f \equiv \frac{\partial f^{\prime}}{\partial a_{2}}+3 a_{2} \frac{\partial f}{\partial a_{3}}+\left(3 a_{2}^{2}+4 a_{3}\right) \frac{\partial f}{\partial a_{4}}=0, \\
& U_{8} f \equiv \frac{\partial f^{\prime}}{\partial b_{2}}+3 b_{2} \frac{\partial f}{\partial b_{3}}+\left(3 b_{2}^{2}+4 b_{3}\right) \frac{\partial f}{\partial b_{3}}=0 .
\end{aligned}
$$

The solutions of these equations are

$$
\begin{gathered}
a_{3}-\frac{3}{2} a_{2}^{2}=\frac{y_{3}}{y_{1}}-\frac{3}{2}\left[\frac{y_{2}}{y_{1}}\right]^{2} \equiv \% \\
b_{3}-\frac{3}{2} b_{2}^{2} \equiv \frac{z_{3}}{z_{1}}-\frac{3}{2}\left[\frac{z_{2}}{z_{1}}\right]^{2} \equiv \beta, \\
a_{4}-4 a_{2} a_{3}+3 a_{2}{ }^{3}-\frac{y_{1}}{y_{1}}-4 \frac{y_{2} y_{3}}{y_{1}{ }^{2}}+3\left[\frac{y_{2}}{y_{1}}\right]^{3} \equiv \frac{d a}{d x} \equiv \alpha_{1}, \\
b_{4}-4 b_{2} b_{3}+3 b_{2}{ }^{3} \equiv \frac{z_{1}}{z_{1}}-4 \frac{z_{2} z_{3}}{z_{1}{ }^{2}}+3\left[\frac{z_{2}}{z_{1}}\right]^{3} \equiv \frac{d \beta}{d x}-\beta_{1} .
\end{gathered}
$$

Iutroduce these solutions, as new variables, into $U_{1} f$ and $U_{f_{1}} f$; since

$$
\begin{array}{llll}
U_{1} \alpha=2 \alpha, & U_{1} \alpha_{1}=3 \alpha_{1}, & U_{1} \beta=2 \beta, & U_{1} \beta_{1}=3 \beta_{1}, \\
U_{4} \alpha=0, & U_{4} \alpha_{1}=2 \%, & U_{4} \beta=0, & U_{4} \beta_{1}=2 \beta,
\end{array}
$$

we have the equations

$$
\begin{aligned}
& U_{g_{1}} f \equiv 2 \alpha \frac{\partial f}{\partial \alpha}+2 \beta \frac{\partial f}{\partial \beta}+3 \alpha_{1} \frac{\partial f}{\partial \alpha_{1}}+3 \beta_{1} \frac{\partial f}{\partial \beta_{1}}=0, \\
& \sigma_{10} f=\alpha \frac{\partial f}{\partial \alpha_{1}}+\beta \frac{\partial f}{\partial \beta_{1}}=0 .
\end{aligned}
$$

The solutions of $\sigma_{10} f$ are

$$
\alpha, \beta, \alpha \beta_{1}-\alpha_{11} \beta \equiv \varphi .
$$

Finally, introduce these solutions, as new variables, into $U_{9} f$; $\operatorname{since}$

$$
U_{9} \varphi=5 \varphi, U_{9} \%=2 \alpha, U_{9} \beta=2 \beta,
$$

we have the equation

the solutions of which are

$$
U_{11} f=2 \alpha \frac{\partial f}{\partial \alpha}=2 \beta \frac{\partial f}{\partial \beta}+5 \varphi \frac{\partial f}{\partial \varphi}=0
$$

$$
\frac{\beta}{\alpha}, \frac{\varphi}{\alpha^{5 / 2}} \equiv \frac{\alpha \beta_{1}-\alpha_{1} \beta}{\alpha^{5 / 2}}, \frac{\varphi}{\beta^{5 / 2}} \equiv \frac{\alpha \beta_{1}-\alpha_{1} \beta}{\beta^{5 / 2}} .
$$

Two, and only two, of these solutions are independent; for, it is clear that the first is a function of the ratio of the last two. 
ROBERTS. ON THE TRANSFORMATION GROUP, E'TC.

18. Having found the two Differential Invariants of the fourth order, we shall now, by means of the Differential Parameter, find the Differential Invariants of the fifth, sixth, seventh, and eighth orders.

Let

$$
\begin{aligned}
& \varphi_{4}=\frac{\alpha \beta_{1}-\alpha_{1} \beta}{\alpha_{1}^{5 / 2}}, \\
& \psi_{4} \equiv \frac{\alpha \beta_{1}-\alpha_{1} \beta}{\beta^{5 / 2}},
\end{aligned}
$$

then

$$
\begin{aligned}
& \Delta \varphi_{1} \equiv \frac{1}{\alpha^{1 / 2}} \frac{d \varphi_{4}}{d x} \equiv \frac{\alpha^{2} \beta_{2}-\alpha \alpha_{2} \beta-\frac{5}{2} \alpha \alpha_{1} \beta_{1}+\frac{5}{2} \alpha_{1}^{2} \beta}{\alpha^{4}}, \\
& \Delta \varphi_{4} \equiv \frac{1}{\alpha^{1 / 2}} \frac{d \varphi_{4}}{d x} \equiv \frac{\alpha \beta \beta_{2}-\alpha_{2} \beta^{2}-\frac{5}{2} \alpha_{1} \beta_{1}^{2}+\frac{5}{2} \alpha_{1} \beta \beta_{1}}{\alpha^{1 / 2} \beta_{7^{7}}^{2}},
\end{aligned}
$$

are independent Differential Invariants of the fifth order.

If we multiply the tirst by $\frac{\beta}{\alpha}$ and the second by $\frac{\alpha \beta^{7 / 2}}{\alpha^{9 / 2}}$, the results will still be independent Differential Invariants of the fifth order, since $\frac{\beta}{\alpha}$, and every function of $\frac{\beta}{\alpha}$, is an Invariant.

Adding together these results, we have

$$
\frac{2 \beta \beta_{2}-\frac{5}{2} \beta_{1}^{2}}{\alpha^{3}}-\frac{\beta^{2}\left[2 \alpha \alpha_{2}-\frac{5}{2} \alpha_{1}^{2}\right]}{\alpha^{5}} .
$$

Multiplying this by $\frac{2 a^{3}}{\beta^{3}}$, we get

$$
\frac{4 \beta \beta_{2}-5 \beta_{1}^{2}}{\beta^{3}}-\frac{\alpha}{\beta} \frac{4 \alpha \alpha_{2}-5 \alpha_{1}^{2}}{\alpha^{3}}
$$

hence the Differential Invariants of the fifth order may be written

$$
\varphi_{5} \equiv \frac{4 \alpha \alpha_{2}-5 \alpha_{1}^{2}}{\alpha_{0}^{3}}, \quad \psi_{5} \equiv \frac{4 \beta \beta_{2}-5 \beta_{1}^{2}}{\beta^{3}},
$$

the first of which, $\varphi_{i}$, contains $y_{i}$ only, and the second, $\phi_{i}$, contains $z_{t}$ only.

If we multiply the Differential Parameter, $\Delta \varphi \equiv \frac{1}{\alpha^{1 / 2}} \frac{d \varphi}{d * c}$ by $\left[\frac{\alpha}{\beta}\right]^{1 / 2}$ we get $\Delta^{\prime} \varphi \equiv \frac{1}{\beta^{1 / 2}} \frac{d \varphi}{d x}$, which is also a Differential Parameter, since it is a function of $\frac{\beta}{\alpha}$ and $\Delta \varphi(\$ 15)$. 
We can now write down the Differential Invariants of the sixth order;

$$
\begin{aligned}
& \varphi_{6} \equiv \frac{1}{\alpha_{0}^{1 / 2} \frac{d \varphi_{5}}{d x}}=\frac{4 \alpha^{2} \alpha_{3}-18 \alpha \alpha_{1} \alpha_{2}+15 \alpha_{1}^{3}}{\alpha^{9 / 2}}, \\
& \varphi_{0} \equiv \frac{1}{\beta^{1 / 1^{2}}} \frac{d \psi_{5}}{d x}=\frac{4 \beta^{2} \beta_{3}-18 \beta \beta_{1} \beta_{2}+15 \beta_{1}^{3}}{\beta^{3 / 2}} ;
\end{aligned}
$$

of the seventh order ;

$$
\begin{aligned}
& \varphi_{7} \equiv \frac{1}{d^{1 / 2}} \frac{d \varphi_{6}}{d x}=\varphi_{6} \frac{d \varphi_{6}}{d \varphi_{5}}, \\
& \psi_{7} \equiv \frac{1}{\beta^{1 / 2}} \frac{d \psi_{6}}{d x}=\varphi_{0} \frac{d \dot{\varphi}_{6}}{d \psi_{5}}
\end{aligned}
$$

finally, of the eighth order ;

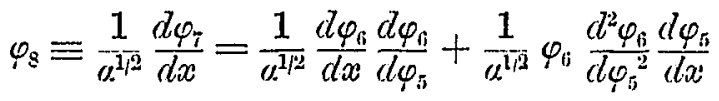

$$
\begin{aligned}
& \equiv \varphi_{6}\left[\frac{d \varphi_{0}}{d \varphi_{5}}\right]^{2}+\varphi_{6} \frac{d^{2} \varphi_{6}}{d \varphi_{5}{ }^{2}}
\end{aligned}
$$

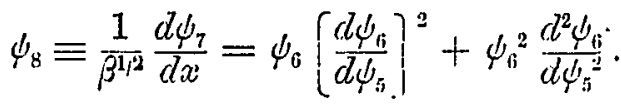

The $\varphi_{i}$ contain $y_{i}$, only, and are, therefore, invariant under the Sub-Group

$$
p, \quad q, \quad x p, \quad y q, \quad x^{2} p, \quad y^{2} q
$$

and the $\phi_{i}$ contain $z_{i}$ only, and are, consequently, invariant under the SubGroup

$$
p, \quad r, \quad x p, \quad z r, \quad x^{2} p, \quad z^{2} r
$$

19. To Find what Differential Equations are Invariant under the $G_{9}$.

In order to find the invariant differential equations of the $n$th order, which are independent of the Differential Invariants, we have to form the matrix, or determinant, of the coefficients of $p, q, r, p_{i}, q_{i}(i=1,2, \ldots, n)$ occurring in the simultaneous system obtained by equating to zero ench transformation extended $n$ times. Having formed the matrix, or determinant, we have to find what equations $f\left(x, y, z, y_{i}, z_{i}\right)=0$ make all the determinants of the matrix, or all the minors of the determinant, zero simultaneously.

By examining the matrices, or determinant, it may readily be shown that 
there are no differential equations, of an order lower than the third, invariant under the $G_{0}$, except those obtained from the Differential Invariant of the third order.

(a). Since the Differential Invariant of the third order does not contain $x, y$, or $z$, there are no differential equations of the zero order invariant under the $G_{9}$.

(b). If we put the Differential Invariant of the third order,

$$
\frac{\beta}{a}=\frac{y_{1}^{2}\left(2 z_{1} z_{3}-3 z_{2}^{2}\right)}{z_{1}^{2}\left(2 y_{1} y_{3}-3 y_{2}^{2}\right)}
$$

first equal to zero, and then equal to infinity, we obtain the following invariant differential equations of the first order

$$
y_{1}=0, \frac{1}{z_{1}}=0, z_{1}=0, \frac{1}{y_{1}}=0 .
$$

These together with the equation $x_{1}=0$ (which, of course, is invariant) are the only differentil equations of the first order invariant under the $G_{\mathrm{a}}$.

(c). It is cl ar that there are no differential equations of the second order invariant under the $G_{i !}$.

The two equations, of the second order,

$$
y_{2}=0, \quad z_{2}=0,
$$

are invariant under the $G_{9}$, only when they are considered in connection with and as a consequence of $y_{1}=0$, and $z_{1}=0$. Hence they are excluded in this discussion.

(d). If we put the Differential Invariant of the third order,

$$
\frac{\beta}{\varkappa} \equiv \frac{y_{1}^{2}\left(2 z_{1} z_{3}-3 z_{2}^{2}\right)}{z_{1}{ }^{2}\left(2 y_{1} y_{3}-3 y_{2}{ }^{2}\right)}
$$

first equal to zero and then equal to infinity, we obtain the following invariant differential equations, of the third order,

$$
\begin{aligned}
& 2 z_{1} z_{3}-3 z_{2}^{2}=0, \\
& 2 y_{1} y_{3}-3 y_{2}^{2}=0 .
\end{aligned}
$$

These are the only two independent differential equations of the third order invariant under the $G_{0}$.

(e). The invariant differential equations of higher orders are obtained by putting some arbitrary function of the Differential invariants equal to zero. 


\section{III.}

\section{Equrvalence of Curves.}

20. In this section we shall make use of the Differential Invariants and invariant differential equations found in the last section, in order to determine the nature of the curve-families which are composed of those curves which are "equivalent" by means of the Group.

21. Two curves are said to be "equivalent" by means of a Group, if, by means of the transformations of the Group, the one curve can be carried over into the other.

Suppose a curve is subjected to the transformations of a Group of $r$ parameters; it will then assume $\infty^{r}$ different positions, provided that it is not invariant under (or, as we sometimes say, does not "admit of ") any one of the $r$ transformations of the Group. In this case, there is generated a family consisting of $\infty^{\prime \prime}$ curves; and it is clear. that curves, which admit of no transformation of the $G$, and which are equivalent, must belong to such a family. If the original curve admits of $q$ independent infinitesimal transformations of the $G_{r}$, it is readily seen that the resulting family will consist of $\infty^{r-q}$ curves; aud these $\infty^{r-4}$ curves are, eo ipso, equivalent among each other by means of the $G_{, .}$.

It is evident that such a curve-family is invariant, where invariant is taken in the sense that the curves of the family are interchanged among ench other, while the family as a whole is unchanged.

This family is defined by two independent differential equations, one of the mith orider and one of the nth order, where $m+n=r-q$, which as a system is invariant under the $G_{9}^{\gamma}$.

22. Since the curve-family, to which the equivalent curves belong, is defined by an invariant system of differential equations, we shall, as a matter of convenience, put here those invariant systems of differential equations, which we shall need in the discussion of this subject.

The most general invariant differential equations are (see $\$ 19)$ :

0. O. None,

I. $O . y_{1}=0, z_{1}=0, x_{1}=0, \quad(\operatorname{see} \S 19, \mathrm{~b})$

II. O. None,

III $O . \psi_{3}=$ const., $2 z_{\mathrm{i}} z_{3}-3 z_{1}{ }^{2}=0,2 y_{1} y_{3} \ldots .3 y_{1}{ }^{2}=0, \quad$ (see 19, d)

IV. $O . \quad \Omega\left(\psi_{3}, \psi_{4}, \varphi_{4}\right)=0$, 
V. $O . \quad \Omega\left(\psi_{3}, \psi_{4}, \phi_{5}, \varphi_{4}, \varphi_{5}\right)=0$,

VI. $O . \quad Q\left(\phi_{3}, \ldots, \phi_{6}, \varphi_{4}, \varphi_{5}, \varphi_{6}\right)=0$,

VII. $0 . \quad Q\left[\psi_{3}, \ldots, \varphi_{0}, \frac{d \psi_{6}}{d \psi_{5}}, \varphi_{1}, \ldots, \frac{d \varphi_{6}}{d \varphi_{5}}\right]=0$

VIII. $0 . \quad Q\left[\psi_{3}, \ldots, \frac{d \psi_{6}}{d \psi_{5}}, \frac{d^{2} \psi}{d \varphi_{5}^{2}}, \varphi_{4}, \ldots, \frac{d^{2} \varphi_{i}}{d \varphi_{5}^{2}}\right]=0$

We can now write down the invariant systems of differential equations, which must consist of one differential equation of the $m$ th order and one of the $n$th order, where $m+n=9-q$, and where $q$ is the number of independent infinitesimal transformations of which the curve admits.

There are three invariant differential equations of the first order ; but, on account of the symmetry of the Group, we may clearly choose $z_{1}=0$ as the typical invariant differential equation of the first order. Now when $\approx_{1}=0$, it is readily seen that $\psi_{3}, \psi_{4}, \psi_{5}, \psi_{0}, \frac{d \psi_{6}}{d \psi_{\bar{j}}}, \frac{d^{2} \psi_{6}}{d \psi_{5}^{2}}$, and $\varphi_{4}$ become zero, so that the invariant systems containing $z_{1}=0$ are :

I. and 0.0 . None,

I. and

I. $O .\left\{\begin{array}{l}z_{1}=0, \\ y_{1}=0,\end{array}\right.$

I. and

II. O. None,

I. and III. $O .\left\{\begin{array}{l}s_{1}=0, \\ 2 y_{1} y_{3}-3 y_{1}{ }^{2}=0,\end{array},(\right.$ See $\$ 19, d)$

I. and IV. O. None,

I. and V. O. $\left\{\begin{array}{l}z_{1}=0 \\ \varphi_{5}=\text { const. }\end{array}\right.$

I. and VI. $O .\left\{\begin{array}{l}z_{1}=0, \\ \varphi_{6}=f\left(\varphi_{5}\right),\end{array}\right.$ 


$$
\begin{aligned}
& \text { I. and VII. O. }\left\{\begin{array}{l}
z_{1}=0, \\
\Omega\left[\varphi_{5}, \varphi_{0}, \frac{d \varphi_{i}}{d \varphi_{5}}\right]=0,
\end{array}\right.
\end{aligned}
$$

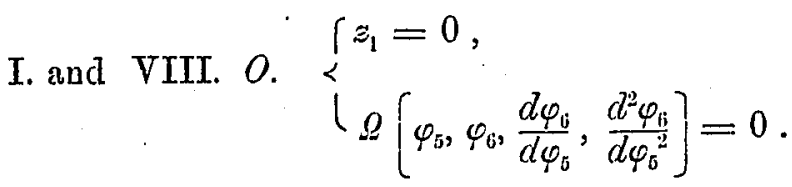

As there is no invariant differential equation of the second order, there are, evidently, no inviriant systems containing a differential equation of the secoud order.

If $\psi_{3}=$ const., it is evident that $\psi_{4}, \psi_{5}, \phi_{6}$, and $\varphi_{4}$ are identically zero, so that the invariant systems containing a differential equation of the third order are:

$$
\begin{aligned}
& \text { III. and III. O. }\left\{\begin{array}{l}
2 z_{1} z_{3}-3 z_{2}{ }^{2}=0, \\
2 y_{1} y_{3}-3 y_{2}{ }^{2}=0,
\end{array} \quad\right. \text { (See 19, d) } \\
& \text { III. and IV. } O \text {. None, } \\
& \text { III. and V. O. }\left\{\begin{array}{l}
\phi_{\mathrm{s}}=\text { const., } \\
\psi_{\mathrm{s}}=\text { const. }
\end{array}\right. \\
& \text { III. and VI. O. }\left\{\begin{array}{l}
\varphi_{3}=\text { const., } \\
\varphi_{6}=f\left(\varphi_{5}\right)
\end{array}\right.
\end{aligned}
$$

The invariant systems containing an invariant differential equation of the fourth order are

$$
\begin{aligned}
& \text { IV. and IV. } O .\left\{\begin{array}{l}
Q_{1}\left(\psi_{3}, \psi_{4}, \varphi_{4}\right)=0, \\
Q_{2}\left(\psi_{3}, \psi_{4}, \varphi_{4}\right)=0,
\end{array}\right. \\
& \text { IV. and V. } O .\left\{\begin{array}{l}
Q\left(\psi_{3}, \psi_{4}, \varphi_{4}\right)=0, \\
\Omega\left(\psi_{3}, \psi_{4}, \psi_{5}, \varphi_{4}, \varphi_{5}\right)=0 .
\end{array}\right.
\end{aligned}
$$

23. We shall now show how to find the curve-families to which those curves belong that are equivalent by meaus of the Group of nine parameters

$$
p, \quad g, \quad r, \quad x p, \quad y q, \quad z r, \quad x^{2} p, \quad y^{2} q, \quad z^{2} r
$$


I. If the curve admits of no infinitesimal transformation of the $G_{9}$, it will generate a fumily of ${ }^{9}{ }^{9}$ curves, when subjected to all the transformations of the $G_{0}$.

(a). This family might be clefined by an invariant system cousisting of one differential equation of the zero order and one of the ninth order; but, as there is no invariant system containing a differential equation of the zero order, this case is excluded.

(b). This family may be defined by the invariant system

$$
\begin{aligned}
& \approx_{1}=0 \\
& \varrho\left[\varphi_{i j}, \varphi_{i j}, \frac{d \varphi_{i j}}{d \varphi_{i j}}, \frac{d^{2} \varphi_{i j}}{d \varphi_{i}^{2}}\right]=0,
\end{aligned}
$$

which consists of one differential equation of the first order and one of the eighth order.

Evidently the curves of this invariant fimily are plane curves in the planes $z=$ const. Since $z r$ and $z^{2} r$ change nothing in the ay-plane, these $\infty^{s}$ plane curves are the same in each plane $z=$ const.; hence, in the $x y$-plane they are defined by the invariant differential equation

$$
Q\left[\varphi_{i,}, \varphi_{i}, \frac{d \varphi_{0}}{d \varphi_{i}}, \frac{d^{2} \varphi_{i j}}{d \varphi_{i}^{2}}\right]=0
$$

which is of the eighth order, anch is griven in terms of $y_{1}, \ldots, y_{s}$.

(c). This family might be defined by an invariant system containing one differential equation of the second order and one of the seventh order; but, as there is no invariant system containing a differentiul equation of the second order, this case is excluded.

(d). The family may be defined by the invariant system

$$
\begin{aligned}
& \psi_{3}=\text { const. } \\
& \varphi_{i}=f\left(\varphi_{i}\right),
\end{aligned}
$$

which consists of one differential equation of the thim order and one of the sixth order.

If $\phi_{3}=0$, the fimily will be defined by the invariant system

$$
\begin{gathered}
2 \approx_{1} z_{3}-3 \approx_{2}{ }^{2}=0, \\
\varphi_{6}=f\left(\varphi_{5}\right) .
\end{gathered}
$$

The integral of the first of these equations is

$$
x z+a_{1} \dot{x}+b_{1} z+c_{1}=0,
$$


and the curves of the family will then be the intersections of the hyperbolic cylinders represented by this equation, and the cylinders given by the equation

$$
\varphi_{i}=f\left(\varphi_{s}\right) \text {. }
$$

If $\psi_{3}=\infty$, then $\% \equiv \frac{y_{3}}{y_{1}}-\frac{3}{2}\left[\frac{y_{2}}{\frac{1}{1}}\right]^{2}=0 ;$ and, consequently, $\varphi_{i j}$ and $\varphi_{5}$ become identically zero; hence this case is excluded.

(e). This family may be defined by the invariant system

$$
\begin{aligned}
& \Omega\left(\psi_{3}, \psi_{4}, \varphi_{4}\right)=0, \\
& \varrho\left(\psi_{3}, \psi_{4}, \psi_{5}, \varphi_{4}, \varphi_{5}\right)=0,
\end{aligned}
$$

which consists of one differential equation of the fourth order aud one of the fifth order.

II. If the curve admits of one of the infinitesimal transformations of the $G_{3}$, it will generate a family of $\omega^{8}$ curves, when subjected to the transformations of the $G_{1}$.

(a). The tirst possible case is that this family may be defined by the invariant system

$$
\begin{gathered}
z_{1}=0, \\
Q\left[\varphi_{5}, \varphi_{i}, \frac{d \varphi_{i j}}{d \varphi_{5}}\right]=0,
\end{gathered}
$$

which consists of one differential equation of the first order and one of the seventh orler. As in $\mathrm{I}$, (b), the curves are plane curves in the plaues $z=$ coust.

(b). The next possible case is that this family may be defined by the invariant system

$$
\begin{aligned}
& \psi_{3}=\text { const. } \\
& \varphi_{5}=\text { const. }
\end{aligned}
$$

which consists of one differential equation of the third order and one of the fifth order.

The same reasoning holds here, as in I, (d), when $\psi_{3}$ becomes either zero or infinity.

(c). This family may be defined by the invariant system

$$
\begin{aligned}
& \Omega_{1}\left(\psi_{3}, \psi_{4}, \varphi_{4}\right)=0, \\
& \Omega_{2}\left(\psi_{3}, \psi_{4}, \varphi_{4}\right)=0,
\end{aligned}
$$

which consists of two differential equations of the fourth order. 
III. If the curve admits of two of the infinitesimal transformations of the $G_{9}$, it will generate a fiemily of $\omega^{\top}$ curves when subjected to the transformations of the $G_{9}$.

The only system invariant under the $G_{9}$, which will define this curvefamily is

$$
\begin{aligned}
& z_{1}=0, \\
& \varphi_{0}=f\left(\varphi_{5}\right),
\end{aligned}
$$

which consists of one differential equation of the first order and one of the sixth order. As in I, (b), the curves are plane curves in the planes $z=$ const.

IV. If the curve admits of three of the infinitesimal transformations of the $G_{y}$, it will generute a fumily of $\infty^{6}$ curves when subjecterl to the transformations of the $G_{9}^{\prime}$.

(a). This family may be defined by the invariant system

$$
\begin{aligned}
z_{1} & =0, \\
\varphi_{i} & =\text { const. }
\end{aligned}
$$

which consists of oue differential equation of the first order and one of the fifth order. As in $I,(b)$, the curves are plane curves in the plane $z=$ const.

(b). This family may be defined by the invariant system

$$
\begin{aligned}
& 2 z_{1} z_{3}-3 z_{2}^{2}=0, \\
& 2 y_{1} y_{3}-3 y_{2}^{2}=0,
\end{aligned}
$$

which consists of two differential equations of the third order.

The integrals of these equations are

$$
\begin{aligned}
& x z-a_{1} x+b_{1} z+c_{1}=0, \\
& x y+a_{2} x+b_{2} y+c_{2}=0 ;
\end{aligned}
$$

hence the $\infty{ }^{6}$ curves are, in this case the intersections of the two families of hyperbolic cylinders, given by the above equations.

V. If the curve admits of four of the infinitesimal transformations of the $G G_{9}$, it will generate a family of ${ }^{5}$ curves when subjected to the transformations of the $G_{9}$.

There is no system invariant under the $G_{3}$, that will define this family of $\infty^{5}$ curves. Hence no such invariant family exists.

VI. If the curve admits of five of the infinitesimal transformations of the $G_{9}$, it will generate a family of $\omega^{4}$ curves when subjected to the transformations of the $G_{0}$. 
The only system invariant under the $G_{9}$, which will define this family is

$$
\begin{aligned}
z_{1} & =0, \\
2 y_{1} y_{3}-3 y_{2}^{2} & =0,
\end{aligned}
$$

which consists of one differential equation of the first order and one of the third order. As in I, (b), the curves will be plane curves in the plaues $z=$ const.

The integral of the equation, $2 y_{1} y_{3}-3 y_{2}^{2}=0$ is

$$
x y+a_{2} x+b_{2} y+c_{2}=0
$$

hence the curves are hyperbolas in the plines $z=$ const.

VII. If the curve admits of six of the infinitesimal transformations of the $G_{3}$, it will generate a family of $\omega^{3}$ curves when suljected to the transformations of the $G_{9}$.

There is no system invariant under the $G_{y,}$, which will define this family of $\infty^{3}$ curves. Hence no such invariant family exists.

VIII. If the curve almits of seven of the infinitesimal transformations of the $G_{0}$, it will generate " $f^{\prime}$ amily of $\omega^{2}$ curves when subjected to the trinstormations of the $G_{9}$.

The typical system invariant under the $G_{10}$ which will define this family is

$$
\begin{aligned}
& z_{1}=0, \\
& y_{1}=0,
\end{aligned}
$$

which consists of two differential equatious of the first order.

In this case, it is evident that the curve-fimily consists of the $\infty^{2}$ straight lines perpendicular to the $x: z$-plane.

IX. If the curve almits of eight of the infinitesimal trensformations of the $G_{9}$, it will generate a fumily of on aurves when subjected to the transformations of the $G_{\mathrm{g}}$.

There is no system invariant uncler the $G_{9}$ that will define this family of $\infty^{1}$ curves; consequently, no such invariant fumily exist.

Hence, if the two curves are equivalent by means of the transformations of the $G_{n}$, they must both belong to some of the families defined above. When the equations to the two curves are given, we can substitute in the types of differential equatious defiving the above invariant families, and if such differential equations are satisfied by the equations of both curves, the curves belong to that family and are equivalent. 
ROBERTS. ON THE TRANSFORMATION GROUP, ITTC.

24. Lie has developed a theory of integration, which may be applied to the integration of some of the above differential equations. We shall, therefore, give here two of his theorems."

I. "If a differential equation of the $m$ th order admits of the Group

$$
q, \quad y q, \quad y^{2} q, \quad p, \quad x p, \quad x^{2} p
$$

it is reducible to the form

$$
Q\left[\varphi_{i}, \varphi_{b}, \ldots, \frac{a^{m-i} \varphi_{6}}{d \varphi_{b}^{m-i}}\right]=0
$$

where

$$
\begin{aligned}
& \varphi_{s}=4 \alpha_{2}-5 \mu_{1}^{2} \\
& \varphi_{6}=\frac{4 \alpha^{2} \alpha_{2}-18 \alpha_{2} \alpha_{1} \alpha_{2}+15 \alpha_{1}^{3}}{\left.\alpha_{1}^{9}\right)^{2}},
\end{aligned}
$$

and

$$
u=\frac{y_{3}}{y_{1}}-\frac{3}{2}\left[\begin{array}{l}
y_{2} \\
y_{1}
\end{array}\right]^{2}, \quad \mu_{1}=\frac{d a}{d x}, \ldots
$$

By integrating the equation of the $(m-6)$ th order, $Q=0$, we get a relation

$$
\varphi_{0}=f\left(\varphi_{i}\right)
$$

which is a differential equation of the third order in $x$ and $\alpha$. This equation admits of the three known infinitesimal transformations $p, x p, x^{2} p$, which, in the variables $x$ and $\alpha$, have the forms

$$
\frac{\partial f}{\partial x}, \quad x \frac{\partial f}{\partial x}-2 x \frac{\partial f}{\partial x}, x^{2} \frac{\partial f}{\partial x}+4 x u \frac{\partial f}{\partial x} .
$$

In order to integrate the above differential equation of the third order, we introduce as new variables the quantities

then

$$
u=\alpha^{1 / 2} \alpha_{1}, \quad \varphi_{i}=4 u^{-2} \alpha_{-2}-5 \alpha^{-3} \alpha_{1}^{2}
$$

$$
\frac{d u}{d \varphi_{5}}=\frac{\varphi_{i}-u^{2}}{\varphi_{i}} \text {. }
$$

If $W\left(u, \varphi_{5}\right)=$ const., is an integral of this Riccatian equation of the first order, we find, in the following manner, by mere differentiation, the other two integral equations of the differential equation $\varphi_{i}=f^{\prime}\left(\varphi_{i}\right)$.

* Math. Annalen, Ba. XXXII, pp. 262, 260.

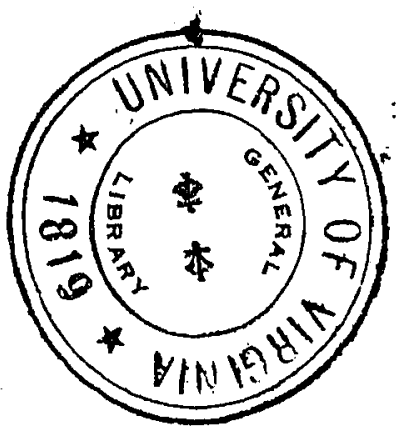


If we put

$$
\nabla f^{\prime} \equiv x^{2} \frac{\partial f}{\partial x}-4 x \alpha^{\prime} \frac{\partial f}{\partial \mu_{1}}-\left(6 x \alpha_{1}+4 \alpha\right) \frac{\partial f}{\partial u_{1}}-\left(8 x \alpha_{2}+10 \alpha_{1}\right) \frac{\partial f}{\partial u_{2}},
$$

then

$$
\begin{aligned}
U_{\varphi_{1}} & =0, \\
U W & =\frac{\partial f}{\partial u} U_{u}=-4 \frac{\partial W}{\partial u} \alpha^{-\zeta_{1}}, \\
U U W & =16 \frac{\partial^{2} W}{\partial u^{2}} u^{-1}-8 x \alpha^{-1 / s} \frac{\partial W}{\partial u} ;
\end{aligned}
$$

and since the quantities $W, U W$, and $U U \cdot W$ are independent as regards $u$, $\varphi_{i}, x$, and $\alpha$, by eliminating the quantities in $u$, and $\varphi_{5}$, between the three equations

$$
W=\text { const. }, \quad \sigma W=\text { const. } \quad U U W=\text { const. }
$$

we find the quantity $a$ determined as a function of $x$ in the form

$$
\%=F(x) \text { : }
$$

This equation is a differential equation of the third order in $x$ and $y . "$

II. "If a differential equation of the mth order admits of the Group.q, $y q, y^{2} q$, it can be reduced to the form

where

$$
Q\left[x, u, \frac{d \alpha}{d x}, \ldots, \frac{d^{n-3} u}{d x^{m+m}}\right]=0
$$

$$
u=\frac{y_{3}}{y_{1}}-\frac{3}{2}\left[\frac{y_{2}}{y_{1}}\right]^{2}=0
$$

If we integrate this equation of the $(m-3)$ th order, we get a differential equation of the third order of the form

$$
\frac{y_{3}}{y_{1}}-\frac{3}{2}\left[\frac{y_{2}}{y_{1}}\right]^{2}=F(x)
$$

which can be reduced to a Riccatian equation of the first order.

If we put

$$
\frac{y_{2}}{y_{1}}=z
$$

we get

$$
\frac{d z}{d x}=\frac{y_{3}}{y_{1}}-\left[\frac{y_{2}}{y_{1}}\right]^{2}
$$

or

$$
\frac{d z}{d x}=\frac{1}{2} z^{2}+F(x)
$$

If $\theta\left(z_{1} x\right)=$ const. is an integral of this Riccatian equation, we find, in 
ROBER'IS. ON THE TRANSFOMMATION GROUP, EI'C.

the following manner, the other two integral equations of $\alpha=F(x)$, by mere differentiation.

When we put

$$
U f \equiv y^{2} \frac{\partial f}{\partial y}+2 y y_{1} \frac{\partial f}{\partial y_{1}}+\left(2 y y_{2}+2 y_{1}^{2}\right) \frac{\partial f}{\partial y_{2}}
$$

then $U \theta=$ const., and $U U \theta=$ const. are known integrals of the equation $\%=\not r \cdot(x)$.

It is necessary to show that $\theta, U \theta$, and $U U \theta$ are independent functions of $a, y, y_{1}$ and $y_{2}$.

We have

$$
\begin{aligned}
U(x) & =0 \\
U \theta & =\frac{\partial \theta}{\partial z} U_{z}=2 \frac{\partial \theta}{\partial z} y_{1}, \\
U U \Theta & =4 \frac{\partial^{2} \theta}{\partial z^{2}} y_{1}^{2}+4 \frac{\partial \theta}{\partial z} y y_{1} ;
\end{aligned}
$$

so that $\theta, U \theta, U U \theta$ are independent as regards $y, x, y_{1}$ and $z$. Hence the integration of $\alpha=F(x)$ is made to depend upon that of the Riccation equation (1)."

25. The equations

$$
\begin{aligned}
& Q\left[\varphi_{5}, \varphi_{0}, \frac{d \varphi_{i j}}{d \varphi_{5}}, \frac{d^{2} \varphi_{i j}}{d \varphi_{5}^{2}}\right]=0, \\
& Q\left[\varphi_{5}, \varphi_{i j}, \frac{d \varphi_{i j}}{d \varphi_{5}}\right]=0 \\
& \varphi_{0}=f\left(\varphi_{5}\right)=0
\end{aligned}
$$

all admit of the Group

$$
q, \quad y q, \quad y^{2} q, \quad p, \quad x p, \quad x^{2} p
$$

hence the two theorems above may be applied to the integration of these equations as soon as we know the functions $Q$ and $f$.

26. Theorem II may be applied to the integration of the equation

$$
\varphi_{5}=\text { const. }
$$

which may be written in the form

$$
4 \alpha \alpha_{1} \frac{d \alpha_{1}}{d \alpha}-5 \alpha_{1}^{2}-k_{1} \alpha^{3}=0
$$


To integrate this equation, we put $z=\alpha_{1}^{2}$, so that

$$
\frac{d z}{d x}-\frac{5 z}{2} \frac{z}{2}-\frac{\pi_{1}}{z} a^{2}=0
$$

and, hence,

$$
z=\mu_{1}^{2}=k_{1} \alpha^{3}+k_{2} 2^{25 / 2}{ }^{* *}
$$

Solving this equation, we find

the integral of which is

$$
\alpha_{1}=u_{1} \overline{k_{1}{ }^{\prime \prime}+k_{2} \alpha^{12}}
$$

$$
-\frac{4}{k_{2}} \sqrt{\frac{k_{1}^{\alpha^{1 / 2}+k_{2}}}{\alpha^{1 / 2}}}=x+k_{3}
$$

which may be put in the form

or

$$
\%=\left(a^{2} x^{2}+2 b x+c\right)^{-2}
$$

$$
\frac{y_{3}}{y_{1}}-\frac{3}{2}\left[\frac{y_{2}}{y_{1}}\right]^{2}=\left(c^{2} x^{2}+2 b x+c\right)^{-2}
$$

Now by writing $z=\frac{y_{2}}{y_{1}}$, we find

$$
\frac{d z}{d x}=\frac{1}{2} z^{2}+\left(a^{2} x+2 b x+c\right)^{-2} .
$$

Suppose $\theta(z, x)=$ const., is an integral of this Riccatian equation of the first order. If we put

then

$$
U f^{\prime}-y^{2} \frac{\partial f}{\partial y}+2 y y_{1} \frac{\partial f}{\partial y_{1}}+\left(2 y_{2} y_{2}+2 y_{1}^{2}\right) \frac{\partial f}{\partial y_{2}},
$$

$$
\sigma \theta=\text { const., } \quad U U \theta=\text { const. }
$$

are two known integrals of the equation

$$
\frac{y_{3}}{y_{1}}-\frac{3}{2} \cdot\left[\frac{y_{2}}{y_{1}}\right]^{2}=\left(a^{2} x^{2}+2 b x+c\right)^{-2}
$$

and by means of these three integral equations, we can eliminate $y_{1}$ and $y_{2}$, and thus determine the complete integral sought.

27. By means of Theorem II the equation

may be integrated.

$$
2 y_{1} y_{3}-3 y_{2}^{2}=0
$$

Let $z=\frac{y_{2}}{y_{1}} ;$ then

$$
\frac{d z}{d x}=\frac{1}{2} z^{2}
$$


Iutegrating this equation, we find

so that

$$
\theta\left(z_{1} x\right)=z x+k_{1} z+2=0 ;
$$

$$
\begin{gathered}
U \Theta=2 x y_{1}+2 k_{1} y_{1}=k_{3} \\
U U \Theta=2 x y y_{1}+2 k_{1} y y_{1}=k_{3}
\end{gathered}
$$

are two integral equations of the equation

$$
2 y_{1} y_{3}-3 y_{2}^{2}=0
$$

Eliminating $y_{1}$ from the above equations we fiud

$$
x y+a_{2} x+b_{2} y+c_{2}=0 \text {. }
$$

Similarly the integral of the equation.

is

$$
2 z_{1} z_{3}-3 z_{3}^{2}=0
$$

$$
x z+\iota_{1} x+b_{1} z+c_{1}=0 .
$$

28. In case $I,(d), \$ 23$, we have the invariant system

$$
\begin{aligned}
& \psi_{3}=\text { const. } \\
& \varphi_{0}=f\left(\varphi_{5}\right) .
\end{aligned}
$$

Suppose we have integrated the second of these equations and found

$$
\varkappa=F(x) \text {. }
$$

If we substitute this value of $\alpha$ in the equation $\psi_{3}=$ const., we have

$$
\frac{z_{3}}{z_{1}}-\frac{3}{2}\left[\begin{array}{l}
z_{2} \\
z_{1}
\end{array}\right]^{2}=C F(x)
$$

We may now apply Theorem II in order to integrate this equation.

The same reasoning applies to the integration of the invariant system of Case II, (b,) \& 23 .

\section{IV.}

Invariani Partial Differentidal Equations.

29. In this section we shall assume $z$ to be a function of $x$ and $y$, and proceed to find what functions $f(x, y, z, p, q, r, s, t, \pi, 0, \sigma, \tau, \ldots)$ and what equations $f(x, \ldots, \tau, \ldots)=0$ are in variant under the $G_{n}^{r}$,-where

$$
\begin{aligned}
& p=\frac{\partial \hat{z}}{\partial x}, q-\frac{\partial z}{\partial y}, r=\frac{\partial^{2} z}{\partial x^{2}}, \quad s \quad \frac{\partial z}{\partial x \partial y}, t=\frac{\partial^{2} z}{\partial y^{2}},
\end{aligned}
$$

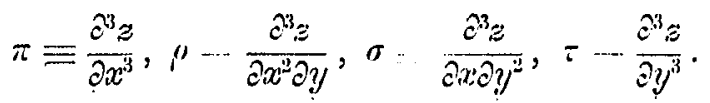


30. To Find what Functions $f\left(x, \ldots, \tau\right.$, are invariant under the $G_{0}$.

If we extend each transformation of the Group in terms of the increments $i p, i q, \ldots, \delta \tau$, and then put each extended transformation equal to zero, we shall have a complete system consisting of nine equations in twelve variables, from which the invariant functions $f$ may be found.

We must first, then, determine the increments $j p, j q, \ldots, j \tau$.

Since $z$ is a function of $x$ and $y$, we have the following identities, which must hold for all values of $x$ and $y$ :

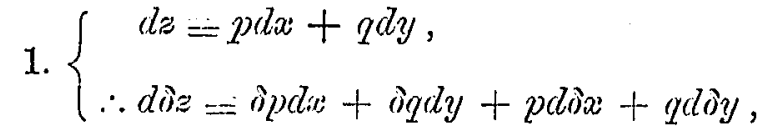

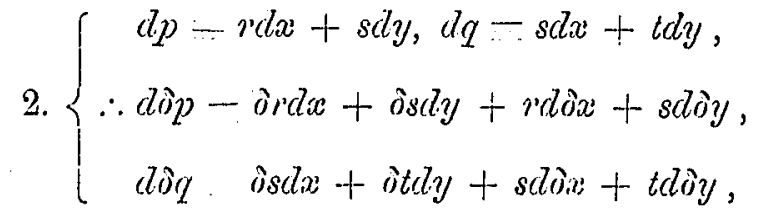

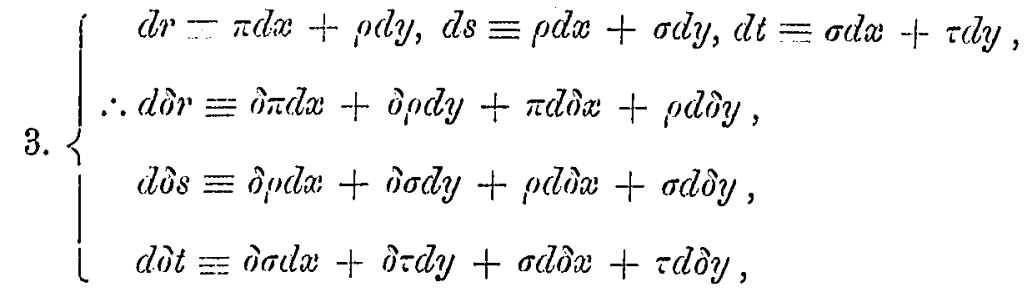

where $\partial x, \partial y$, and $\partial z$ are the coefficients of $\frac{\partial f}{\partial x}, \frac{\partial f}{\partial y}, \frac{\partial f}{\partial z}$, respectively, in each transformation.

In the trinsformation $U f \equiv \frac{\partial f}{\partial x}$, we have $i x=1, i y=i z=0$, so that

$$
\partial p=\partial q=\ldots=i \tau=0 \text {. }
$$

Similarly the trunsformations $U f \quad \frac{\partial f}{\partial y}$ and $U f \equiv \frac{\partial f}{\partial x}$ give

$$
i p=i q=\ldots=i \tau=0 \text {. }
$$

In the transformation $U \equiv x \frac{\partial f}{\partial x}$, we have $\partial x=x, \partial y=0$, $\partial z=0$. Substituting these values of $\partial x, \partial y, i z$ in equations 1 , we have

$$
0 \equiv \lambda i p d x+i g d y+p d l x
$$

so that $i p=-p, \partial q=0$. 
ROBERTS. ON THE TRANSFORMATION GROUP, E'TC.

If we put these values of $\delta p$.and $\partial q$ in equations (2), we get

$$
\begin{gathered}
-d p \equiv-r d x-s d y=\delta r d x+\delta s d y+r d x, \\
0 \equiv \delta s d x+\delta t d y+s d x ;
\end{gathered}
$$

so that $i r^{r}=-2 r, \delta s=-s, i t=0$.

Putting these values of $\delta r, \partial s, \delta t$ in equations (3), we get

$$
\begin{gathered}
-2 d r \equiv-2(\pi d x+\rho d y) \equiv \delta \pi d x+\delta \rho d y+\pi d x, \\
-d s \equiv-\rho d x-\sigma d y \equiv \delta \rho d x+\delta \sigma d y+\rho d x, \\
0 \equiv \delta \sigma d x+\delta \tau d y+\sigma d x ;
\end{gathered}
$$

therefore, $\delta \pi=-3 \pi, \delta_{i} \rho=-2 \rho, i \sigma=-\sigma, i \tau=0$.

Hence, the transformation, $U f \equiv x \frac{\partial f}{\partial x}$, extended becomes

$$
U f=x \frac{\partial f}{\partial x}-p \frac{\partial f}{\partial p}-2 r \frac{\partial f}{\partial r^{\prime}}-s \frac{\partial f}{\partial s}-3 \pi \frac{\partial f}{\partial \pi}-2 \rho \frac{\partial f}{\partial \rho}-\sigma \frac{\partial f}{\partial \sigma} .
$$

If, in a similar manner, we extend the other transformations and put each extended transformation equal to zero, we get the following complete system :

$$
\begin{aligned}
& \frac{\partial f}{\partial x}=0, \frac{\partial f}{\partial y}=0, \frac{\partial f}{\partial z}=0 \text {, } \\
& x \frac{\partial f}{\partial x}-p \frac{\partial f}{\partial p}-2 r \frac{\partial f^{\prime}}{\partial r^{\prime}}-s \frac{\partial f^{\prime}}{\partial s}-3 \pi \frac{\partial f^{\prime}}{\partial \pi}-2 r \frac{\partial f}{\partial f^{\prime \prime}}-\sigma \frac{\partial f^{\prime}}{\partial \sigma}=0 \text {, } \\
& y \frac{\partial f}{\partial y}-q \frac{\partial f}{\partial q}-s \frac{\partial f}{\partial s}-2 t \frac{\partial f}{\partial t}-0 \frac{\partial f}{\partial "}-2 \sigma \frac{\partial f}{\partial \sigma}-3 \tau \frac{\partial f}{\partial t}=0 \text {. } \\
& z \frac{\partial f}{\partial z}+p \frac{\partial f}{\partial p}+q \frac{\partial f}{\partial q}+r \frac{\partial f}{\partial r}+s \frac{\partial f}{\partial s}+t \frac{\partial f}{\partial t}+\pi \frac{\partial f}{\partial \pi}+\sigma \frac{\partial f}{\partial \sigma}+=\frac{\partial f}{\partial t}=0, \\
& x^{2} \frac{\partial f}{\partial x}-2 x p \frac{\partial f}{\partial p}-2(p+2 x r) \frac{\partial f}{\partial r}-2 x s \frac{\partial f}{\partial s}-6(r+\pi x) \frac{\partial f}{\partial \pi} \\
& -2(s+2, x) \frac{\partial f}{\partial f^{\prime \prime}}-2 \sigma x \frac{\partial f}{\partial \sigma}=0 \\
& y^{2} \frac{\partial f}{\partial y}-2 y q \frac{\partial f}{\partial q}-2 s y \frac{\partial f}{\partial s}-2(q+2 t y) \frac{\partial f}{\partial t}-2 m y \frac{\partial f}{\partial f^{\prime}}-2(s+2 \sigma y) \frac{\partial f}{\partial \sigma} \\
& -6(t+y \tau) \frac{\partial f}{\partial t}=0 \\
& z^{2} \frac{\partial f}{\partial z}+2 z p \frac{\partial f}{\partial p}+2 z q \frac{\partial f}{\partial q}+2\left(z r+p^{2}\right) \frac{\partial f}{\partial r^{\prime}}+2(z s+p q) \frac{\partial f}{\partial s} \\
& +2\left(\tau^{2}+z t\right) \frac{\partial f}{\partial t}+2(3 r p+z \pi) \frac{\partial f}{\partial \pi}+2\left(t^{\prime} t+z_{0}+2 x p\right) \frac{\partial f}{\partial p} \\
& +2(2 s q+\sigma z+p t) \frac{\partial f}{\partial \sigma}+3(2 q t+z \tau) \frac{\partial f}{\partial t}=0 .
\end{aligned}
$$


Since the first three equations show that the solutions will be independent of $x, y$, and $z$, we neglect those terms containing $\frac{\partial f}{\partial x}, \frac{\partial f}{\partial y}, \frac{\partial f}{\partial z}$, and then, by algebraic reduction, we obtain

$$
\begin{aligned}
& U_{1} f \equiv p \frac{\partial f}{\partial p}+2 v \frac{\partial f}{\partial t}+s \frac{\partial f}{\partial s}+3 \pi \frac{\partial f}{\partial \pi}+20 \frac{\partial f}{\partial t}+\sigma \frac{\partial f}{\partial \sigma}=0 \\
& U_{2} f=q \frac{\partial f}{\partial q}+s \frac{\partial f}{\partial s}+2 t \frac{\partial f}{\partial t}+r \frac{\partial f}{\partial t}+2 \sigma \frac{\partial f}{\partial \sigma}+3 z \frac{\partial f}{\partial t}=0 \text {, } \\
& U_{3 r} f=p \frac{\partial f}{\partial p}+q \frac{\partial f}{\partial q}+r \frac{\partial f}{\partial t}+s \frac{\partial f}{\partial s}+t \frac{\partial f}{\partial t}+\pi \frac{\partial f}{\partial \pi}+\partial^{\prime} \frac{\partial f}{\partial "}+\sigma \frac{\partial f}{\partial \sigma}+\tau \frac{\partial f}{\partial t}=0, \\
& \cdot U_{\mathrm{v}} f^{\prime}-p \frac{\partial f^{\prime}}{\partial \gamma^{\prime}}+3 r^{\prime} \frac{\partial f^{\prime}}{\partial \pi}+s \frac{\partial f^{\prime}}{\partial \prime^{\prime}}=0 \\
& U_{i} f=\eta \frac{\partial f}{\partial t}+s \frac{\partial f}{\partial \sigma}+3 t \frac{\partial f}{\partial t}=0 \text {, }
\end{aligned}
$$

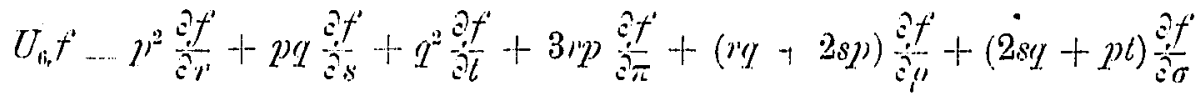

$$
\begin{aligned}
& +3 q t \frac{\partial f}{\partial t}=0
\end{aligned}
$$

This is a complete system of six equations in nine variables.

The matrix formed with the coefficients of $\frac{\partial f^{\prime}}{\partial j}, \ldots, \frac{\partial f^{\prime}}{\hat{z}}$, is

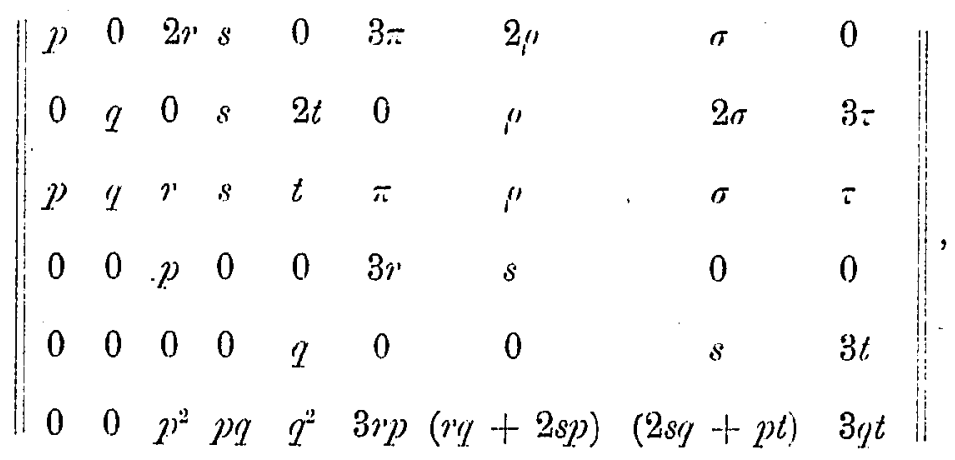

the determinants of which do not vanish identically, so that there cre three, and only three, independent solutions of the complete system.

In order to find these three solutions, we proceed as follows: (See $\$ 13$.) The solutions common to $U_{s_{1}} f$ and $U_{s_{1}} f^{\prime}$ are $3 r^{2}-2 p \pi \mu_{1}, \quad s r-p_{p}-\alpha_{2}, \quad 3 t^{2}-2 q \tau=\alpha_{3}, \quad s t-q \sigma \equiv \alpha_{1}, \quad p, q, \quad s$. 
ROBERTS. ON THE TRANSTOORMATION GROUP, ETC.

If we introduce these solutions, $\alpha_{i}, p, q, s$, as new variables into the equations $U_{1} f, U_{2} f, U_{3} f$, and $U_{0,} f$,-since

$$
\begin{aligned}
& U_{1} \alpha_{1}=4 \mu_{1}, \quad U_{1} \alpha_{2}=3 \mu_{2}, \quad U_{1} \alpha_{3}=0 \quad, \quad U_{1} \alpha_{1}=\mu_{4}, \\
& U_{2} \mu_{1}=0 \quad, \quad U_{2} \mu_{2}=\mu_{2}, \quad U_{2} \mu_{3}=4 \pi_{3}, \quad U_{2} \mu_{1}=3 \mu_{1},
\end{aligned}
$$

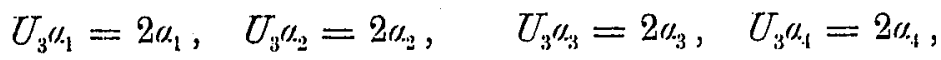

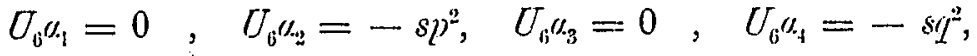

we have the following equations:

$$
\begin{aligned}
& U_{i} f=4 \mu_{1} \frac{\partial f}{\partial u_{1}}+3 u_{-2} \frac{\partial f}{\partial u_{2}}+u_{1} \frac{\partial f}{\partial u_{1}}+p \frac{\partial f}{\partial p}+s \frac{\partial f}{\partial s}=0 \\
& U_{s} f^{\prime} \equiv \alpha_{-2} \frac{\partial f^{\prime}}{\partial \mu_{-2}}+4 \alpha_{3} \frac{\partial f^{\prime}}{\partial \mu_{s}}+3 \alpha_{-1} \frac{\partial f^{\prime}}{\partial \mu_{1}}+2 \frac{\partial f^{\prime}}{\partial q}+s \frac{\partial f^{\prime}}{\partial s}=0 \\
& U_{n} f^{\prime} \equiv 2 \mu_{1} \frac{\partial f^{\prime}}{\partial u_{1}}+2 \mu_{2} \frac{\partial f}{\partial \mu_{2}}+2 \mu_{3} \frac{\partial f}{\partial u_{3}}+2 \mu_{4} \frac{\partial f}{\partial u_{4}}+p \frac{\partial f}{\partial p}+q \frac{\partial f^{\prime}}{\partial q}+s \frac{\partial f}{\partial s}=0 .
\end{aligned}
$$

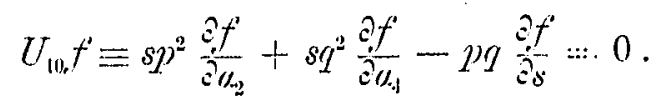

The solutions of $U_{7} f$ are

$$
\frac{\alpha_{1}}{p^{1}} \beta_{1}, \frac{\alpha_{2}}{p^{3}} \equiv \beta_{2}, \frac{\alpha_{4}}{p} \equiv \beta_{3}, \frac{s}{p} \equiv \beta_{4}, \quad \alpha_{3} \cdot q .
$$

If we introduce these solutions, $\beta_{i}, \gamma_{3}, q$, as new variables, into the equatious $U_{\mathrm{s}_{2}} f, U_{0_{2}} f$, and $U_{10_{2}} f$,-since

$$
\begin{aligned}
& U_{s} \beta_{1}=0, \quad U_{s} \beta_{2}=\beta_{2}, \quad U_{8} \beta_{3}=3 \beta_{3}, \quad U_{s} \beta_{4}=\beta_{1}, \\
& U_{9} \beta_{i}=-2 \beta_{1}, \quad U_{9} \beta_{2}=-\beta_{2}, \quad U_{9} \beta_{3}=\beta_{3}, \quad U_{9} \beta_{4}=0, \\
& U_{10} \beta_{1}=0, \quad U_{10} \beta_{2}=\beta_{4}, \quad U_{10} \beta_{3}=\beta_{4} q^{2}, \quad U_{101} \beta_{11}=-q,
\end{aligned}
$$

we have the following equations:

$$
\begin{aligned}
& U_{11} f^{\prime}=\beta_{2} \frac{\partial f}{\partial \beta_{2}}+3 \beta_{3} \frac{\partial f}{\partial \beta_{3}}+\beta_{4} \frac{\partial f}{\partial \beta_{1}}+4 \mu_{3} \frac{\partial f}{\partial \alpha_{3}}+q \frac{\partial f}{\partial g}=0, \\
& U_{12} f^{2}=-2 \beta_{1} \frac{\partial f}{\partial \beta_{1}}-\beta_{2} \frac{\partial f}{\partial \beta_{2}}+\beta_{3} \frac{\partial f}{\partial \beta_{3}}+2 \alpha_{3} \frac{\partial f}{\partial \alpha_{3}}+q \frac{\partial f}{\partial q}=0, \\
& U_{1: f^{\prime}}=\beta_{4} \frac{\partial f}{\partial \beta_{2}}+\beta_{1} g^{2} \frac{\partial f}{\partial \beta_{3}}-q \frac{\partial f}{\partial \beta_{1}}=0 .
\end{aligned}
$$

The solutions of $U_{11} f$ are

$$
\frac{\beta_{2}}{q} \equiv \varphi_{1}, \frac{\beta_{3}}{q^{3}}-\varphi_{2}, \frac{\beta_{4}}{q} \equiv \varphi_{3}, \frac{\alpha_{3}}{q^{i}} \equiv \varphi_{4}, \beta_{1} .
$$


If we introduce these solutions, $\beta_{1}, \varphi_{l}$, as new variables, into the equations $U_{12} f$ and $U_{13} f$, - since

$$
\begin{aligned}
& U_{12}^{*} \varphi_{1}=-2 \varphi_{1}, \quad U_{12} \varphi_{2}=-2 \varphi_{2}, U_{12} \varphi_{3}=-\varphi_{3}, \quad U_{12} \varphi_{1}=-2 \varphi_{11}, \\
& U_{13} \varphi_{1}=\varphi_{3}, \quad U_{13} \varphi_{2}=\varphi_{3}, \quad U_{1:} \varphi_{3}=-1, \quad U_{1: 3} \varphi_{4}=0,
\end{aligned}
$$

we have the following equations :

$$
\begin{aligned}
& U_{11_{1}} \equiv 2 \beta_{1} \frac{\partial f}{\partial \beta_{1}}+2 \varphi_{1} \frac{\partial f}{\partial \varphi_{1}}+2 \varphi_{2} \frac{\partial f}{\partial \varphi_{2}}+\varphi_{3} \frac{\partial f}{\partial \varphi_{3}}+2 \varphi_{1} \frac{\partial f}{\partial \varphi_{1}}=0, \\
& U_{15} f^{\prime} \equiv \varphi_{3} \frac{\partial f}{\partial \varphi_{1}}+\varphi_{3} \frac{\partial f}{\partial \varphi_{2}}-\frac{\partial f}{\partial \varphi_{3}}=0 .
\end{aligned}
$$

The solutions of $U_{15} f$ are

$$
\varphi_{1}-\varphi_{2}=\psi_{1}, \quad 2 \varphi_{1}+\varphi_{3}{ }^{2}, \phi_{2}, \quad \beta_{1}, \varphi_{1} .
$$

If, finally, we introduce these solutions, $\phi_{i}^{\prime}, \beta_{1}, \varphi_{1}$, is new variables, into the equation $U_{1: 1,} f$,-since

$$
U_{14} \psi_{1}=2 \psi_{1}, \quad U_{14} \psi_{2}^{\prime}=2 \psi_{2},
$$

we have the equation

$$
U_{\mathrm{Ii}} f=2 \beta_{1} \frac{\partial f}{\partial \beta_{1}}+2 \psi_{1} \frac{\partial f}{\partial \psi_{1}}+2 \psi_{2} \frac{\partial f}{\partial \psi_{2}}+2 \varphi_{1} \frac{\partial f}{\partial \varphi_{1}}=0
$$

the solutions of which are

$$
\begin{aligned}
& \frac{\beta_{1}}{\varphi_{1}} \equiv \frac{q^{4}\left(3 r^{2}-2 p \pi\right)}{p^{4}\left(3 t^{2}-2 q \tau\right)}, \\
& \phi_{1} \equiv \frac{q^{3}(s r-p o)-p^{2} q(s t-q \sigma)}{p^{3}\left(3 i^{2}-2 \eta \tau\right)}, \\
& \varphi_{4}=\frac{2 q^{2}}{\phi_{1}} \equiv \frac{2 q^{3}\left(s r^{2}-p o\right)+p q^{2} s^{2}}{p^{3}\left(3 t^{2}-2 q^{2}\right)} .
\end{aligned}
$$

These are, therefore, the three independent solutions of the complete system.

31. We shall now Find the Differential Purameter, when $\approx$ is a Finction. of $x$ and $y$.

Suppose $Q\left(z, y, z, p, q, r, \ldots, \varphi, \varphi_{x}, \varphi_{y}\right)$ to be such a function that, when $\varphi$ is an invariant function of the Group, $Q$ is also an invariant function, - to find the function $Q . *$

When we write $\varphi_{x} \equiv \frac{\partial \varphi}{\partial x}$, and $\varphi_{y} \equiv \frac{\partial f}{\partial y}$, we have

$$
i \varphi \equiv \varphi_{x} d x+\varphi_{y} d y
$$

or, since $\varphi$ is an invariant function

$$
d \dot{\varphi} \varphi \equiv 0 \equiv \delta \varphi_{x} d x+\delta \varphi_{y} d y+\varphi_{x} d \lambda x+\varphi_{y} d \dot{\partial} y .
$$

* Sophus Lie, Vurlesungen ueber Contiunierliche Gruppen, puge 670. 
ROBERTS. ON THE TIRANSFORMATION GROUP, ITTC.

From these identities we can determine the increments $\delta \varphi_{x}$ and $\delta \varphi_{y}$ for each transformation of the $G_{0}$.

If we extend each trausformation of the Group in terms of the increments $\partial p, \ldots, \hat{\partial} \pi, \partial \varphi_{x} \delta \varphi_{y}$, and then put each extended transformation equal to zero we obtain the following complete system, from which $Q$ may be determined :

$$
\begin{gathered}
\frac{\partial Q}{\partial x}=0, \frac{\partial Q}{\partial y}=0, \frac{\partial Q}{\partial z}=0, \\
x \frac{\partial Q}{\partial x}-p \frac{\partial Q}{\partial p}-2 r \frac{\partial Q}{\partial r}-s \frac{\partial Q}{\partial s}-3 \pi \frac{\partial Q}{\partial \pi}-\varphi_{x} \frac{\partial Q}{\partial \varphi_{x}}=0, \\
y \frac{\partial Q}{\partial y}-q \frac{\partial Q}{\partial q}-s \frac{\partial Q}{\partial s}-2 \frac{\partial Q}{\partial t}-\varphi_{y} \frac{\partial Q}{\partial \varphi_{y}}=0, \\
z \frac{\partial Q}{\partial z}+p \frac{\partial Q}{\partial p}+q \frac{\partial Q}{\partial q}+r \frac{\partial Q}{\partial r}+s \frac{\partial Q}{\partial s}+t \frac{\partial Q}{\partial t}+\pi \frac{\partial Q}{\partial \pi}=0, \\
x^{2} \frac{\partial Q}{\partial x}-2 x p \frac{\partial Q}{\partial p}-2(p+2 x r) \frac{\partial Q}{\partial r}-2 x s \frac{\partial Q}{\partial s}-6(r+\pi x) \frac{\partial Q}{\partial \pi} \\
y^{2} \frac{\partial Q}{\partial y}-2 y q \frac{\partial Q}{\partial q}-2 y s \frac{\partial Q}{\partial s}-2(q+2 t y) \frac{\partial Q}{\partial t}-2 y \varphi_{y} \frac{\partial Q}{\partial \varphi_{y}}=0 . \\
z^{2} \frac{\partial Q}{\partial z}+2 z p \frac{\partial Q}{\partial p}+2 z g \frac{\partial Q}{\partial q}+2\left(z r+p^{2}\right) \frac{\partial Q}{\partial r}+2\left(q^{2}+z t\right) \frac{\partial Q}{\partial t} \\
+2(z s+p q) \frac{\partial Q}{\partial s}+2(3 r p+z \pi) \frac{\partial Q}{\partial \pi}=0 .
\end{gathered}
$$

By algebraic reduction this complete system may be brought to the form

$$
\begin{aligned}
& U_{1} Q \equiv p \frac{\partial Q}{\partial p}+2 r \frac{\partial Q}{\partial r}+s \frac{\partial Q}{\partial s}+3 \pi \frac{\partial Q}{\partial \pi}+\varphi_{x} \frac{\partial Q}{\partial \varphi_{x}}=0, \\
& U_{2} Q \equiv q \frac{\partial Q}{\partial q}+s \frac{\partial Q}{\partial s}+2 t \frac{\partial Q}{\partial t}+\varphi_{y} \frac{\partial Q}{\partial \varphi_{y}}=0, \\
& U_{3} Q \equiv p \frac{\partial Q}{\partial p}+q \frac{\partial Q}{\partial q}+r \frac{\partial Q}{\partial r}+s \frac{\partial Q}{\partial s}+t \frac{\partial Q}{\partial t}+\pi \frac{\partial Q}{\partial \pi}=0, \\
& U_{1} Q \equiv p \frac{\partial Q}{\partial r}+3 r \frac{\partial Q}{\partial \pi}=0, \\
& U_{5} Q \equiv q \frac{\partial Q}{\partial t}=0 \\
& U_{0} Q \equiv p q \frac{\partial Q}{\partial s}=0 .
\end{aligned}
$$


Equations $U_{5} Q$ and $U_{0} Q$ show that the solutions will be inclependent of $s$ and $t$; hence we neglect those terms containing $\frac{\partial Q}{\partial s}$ and $\frac{\partial Q}{\partial t}$, and the complete system reduces then to one consisting of four equations in six variables.

The matrix formed with the coefficients $\frac{\partial Q}{\partial p}, \frac{\partial Q}{\partial q}, \frac{\partial Q}{\partial r}, \frac{\partial Q}{\partial \tau}, \frac{\partial Q}{\partial \varphi_{x}}, \frac{\partial Q}{\partial \varphi_{y}}$, is

$$
\left|\begin{array}{llllll}
p & 0 & 2 r & 3 \pi & \varphi_{x} & 0 \\
0 & q & 0 & 0 & 0 & \varphi_{y} \\
p & q & r & \pi & 0 & 0 \\
0 & 0 & p & 3 r & 0 & 0
\end{array}\right|,
$$

all the determinants of which do not vanish identically, so that there are two, and only two, inclependent solntions of this complete system.

In order to find these solutions we proceed as follows:

The solutions common to $U_{1} \Omega$ and $U_{2} Q$ are

$$
\frac{r}{p^{2}} \equiv \alpha_{1}, \quad \frac{\pi}{p^{3}} \quad \alpha_{2}, \quad \frac{\varphi_{x}}{p} \ldots a_{3}, \quad \frac{\varphi_{y}}{q}-\alpha_{4} .
$$
since

If we introduce these solutions, $\alpha_{i}$, as new variables, into $U_{3} f$ and $U_{4} f^{\prime}$ -

$$
\begin{aligned}
& U_{3} \mu_{1}=-\mu_{1}, \quad U_{3} \mu_{2}=-2 \alpha_{12}, \quad U_{3} \mu_{3}=-\alpha_{3}, \quad U_{4} \mu_{1}=-\alpha_{1.1}, \\
& U_{4} \alpha_{1}=\frac{1}{p}, \quad U_{4} \alpha_{2}=\frac{3 \alpha_{1}}{p}, \quad U_{3} \alpha_{3}=0, \quad U_{4} \alpha_{4}=0,
\end{aligned}
$$

we have the following equations :

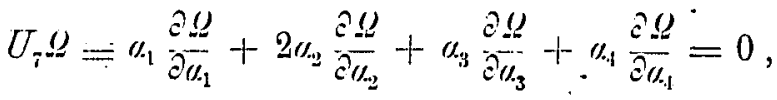

$$
\begin{aligned}
& U_{\mathrm{s}} \equiv \frac{\partial Q}{\partial \tau_{1}}+3 \psi_{1} \frac{\partial Q}{\partial r_{2}}=0 \text {. }
\end{aligned}
$$

The solutions of $U_{8} Q$ are

$$
3 r_{1}^{2}-2 a_{2.2} \equiv \beta, \alpha_{i 3}, \alpha_{4} \text {. }
$$

If we introduce these solutions, $\beta, \alpha_{3}, \alpha_{1}$, as new variables, into the equation $U_{7} Q$,- since

we have the equation

$$
U_{i} \beta=2 \beta,
$$

$$
2 \beta \frac{\partial \dot{Q}}{\partial \beta}+\alpha_{3} \frac{\partial Q}{\partial \alpha_{3}}+\alpha_{1} \frac{\partial Q}{\partial \alpha_{4}}=0,
$$


MOBERIS. ON THE TRANSFORMATION GROUP, EIC.

the solutions of which are

$$
\begin{aligned}
& \mu_{3}^{2}=\frac{p^{2} \varphi_{2}^{2}}{3 r^{2}-2 p \pi}=J_{1} \varphi, \\
& \frac{\alpha_{1}^{2}}{\beta} \equiv \frac{p^{4} \varphi_{y}^{2}}{q^{2}\left(3 r^{2}-2 p \pi\right)} \cdots J_{2} \varphi .
\end{aligned}
$$

$J_{1} \varphi$ and $J_{2} \varphi$ are called Differential Parameters and have the general form

$$
\left.Q(x, y, z, p, \ldots, \varphi,\lrcorner, \varphi,\lrcorner_{2} \varphi\right) .
$$

Since $J_{1} \varphi$ and $J_{2} \varphi$ are invariant functions, the expressions

$$
\begin{aligned}
J_{1}^{2} \varphi & \equiv \Delta_{1}\left(J_{1} \varphi\right) \equiv \frac{\partial J_{1} \varphi}{\partial x}\left\{3 r^{2} \frac{p^{2}}{-2 p \pi}\right\}, \\
J_{2}\left(J_{1} \varphi\right) & \equiv \frac{\partial J_{1} \varphi}{\partial y}\left\{q^{2}\left(3 r^{2}-2 p \pi\right\},\right. \\
J_{1}\left(J_{2} \varphi\right) & \equiv \frac{\partial J_{2} \varphi}{\partial x}\left\{\frac{p^{2}}{3 r^{2}-2 p \pi}\right\}, \\
J_{2}^{2} \varphi & \equiv \frac{\partial J_{2} \varphi}{\partial y}\left\{\frac{p^{1}}{q^{2}\left(3 r^{2}-2 p \pi\right.}\right\},
\end{aligned}
$$

are also Differential Parameters; and, likewise, $J_{1}^{3} \varphi$, etc.; so that the most general Differential Parameter is

$$
\left.\left.\left.\left.\underline{l}(x, y, z, p, \ldots, \varphi,\lrcorner_{1} \varphi,\right\lrcorner_{2} \varphi, J_{1}^{2} \varphi,\right\lrcorner_{2}(\lrcorner_{1} \varphi\right), \ldots\right) \text {. }
$$

By operating with the Differential Parameters on the invariant functions already found, we can find the invariant functions of higher orders.

32. We shall now show how the five invariant functions of the fourth order may be obtained by means of the Differential Parameter.

Let

$$
\begin{aligned}
& \varphi_{1} \equiv \frac{q^{4}\left(3 r^{2}-2 p \pi\right)}{p^{4}\left(3 t^{2}-2 q \tau\right)} \\
& \varphi_{2} \equiv \frac{q^{3}(s r-p \rho)-q p^{2}(s t-q \sigma)}{p^{3}\left(3 t^{2}-2 q \tau\right)} \\
& \varphi_{3} \equiv \frac{2 q^{3}(s r-p \rho)+s^{2} p q^{2}}{p^{3}\left(3 t^{2}-2 q \tau\right)}
\end{aligned}
$$


Hence

$$
\begin{aligned}
& \lrcorner_{1} \varphi_{1}=\frac{p^{2}}{3 r^{2}-2 p \pi}\left[\frac{\partial \varphi_{1}}{\partial x}\right]^{2} \equiv \psi_{1}, \\
& \lrcorner_{1} \varphi_{2} \equiv \frac{p^{2}}{3 r^{2}-2 p \pi}\left[\frac{\partial \varphi_{2}}{\partial x}\right]^{2} \equiv \phi_{2}, \\
& \lrcorner_{1} \varphi_{3}=\frac{p^{2}}{3 r^{2}-2 p \pi}\left[\frac{\partial \varphi_{3}}{\partial x}\right]^{2} \equiv \varphi_{3}, \\
& \lrcorner_{2} \varphi_{1} \equiv \frac{p^{4}}{q^{2}\left(3 r^{2}-2 p \pi\right)}\left[\frac{\partial \varphi_{1}}{\partial y}\right]^{2} \equiv \phi_{4}, \\
& \lrcorner_{2} \varphi_{2} \equiv \frac{p^{4}}{q^{2}\left(3 r^{2}-2 p \pi\right)}\left[\frac{\partial \varphi_{2}}{\partial y}\right]^{2} \equiv \varphi_{5},
\end{aligned}
$$

are five independent functions of the fourth order invariant under the Group. the $G_{0}$.

33. To Find what Equations $f(x, y, z, p, \ldots)=0$ are Invariant under

As in $\$ 19$, it may be shown that, by examining the matrices, or determinaut, of the coefficients of $p, q, r$, etc., there are no partial differential equations of an order lower than the third invariant under the $G_{y}$, except those oblained from the invariant finctions of the third order.

(a). Since none of the invariant functions, $\varphi_{1}, \varphi_{2}, \varphi_{3}$, contain $z$, there are no partial differential equations of the zero order invilriant under the $G_{9}$.

(b). If we put the invariant function of the third order,

$$
\varphi_{1} \equiv \frac{q^{4}\left(3 r^{2}-2 p \pi\right)}{p^{4}\left(3 t^{2}-2 q \tau\right)}
$$

first equal to zero and then equal to infinity, we obtain the following invariant partial differential equation of the first order:

$$
q=0, \quad \frac{1}{p}=0, \quad p=0, \frac{1}{q}=0 .
$$

These are the only partial differential equations of the first order invariant under the $G_{3}$.

(c). It is clear that there are no partial differential equations of the second order invariant under the $G_{9}$; for the equations of the second order,

$$
r=s=t=0
$$

are invariant under the $G_{0}$ only when they are considered in connection with and as a consequence of $p=0$ and $q=0$.

(d). The invariant partial differential equations of higher orders are obtained by putting arbitrary functions of the inyariant functions equal to 
zero. Thus the most general invariaut partial differential equation of the third orcler is

$$
Q\left(\varphi_{1}, \varphi_{2}, \varphi_{3}\right)=0 \text {. }
$$

(e). The most general partial differential equation of the fourth order invariant under the $G_{g}$ is

$$
Q\left(\varphi_{1}, \varphi_{2}, \varphi_{3}, \psi_{1}, \psi_{12}, \psi_{3}, \psi_{4}, \psi_{5}\right)=0 \text {. }
$$

\section{V.}

\section{Enuitalience of Sunfaces.}

34. In this section we shall show how we may determine the nature of the families of surfaces which are composed of those surfaces which are "equivalent" by means of the transformations of the Group.

35. Two surfaces are said to be equivalent by meaus of a Group, if by means of the transformations of the Group, the one surface can be carried over into the other. As in the cuse of equivalent curves, equivalent surfaces beloug to invariant fimilies of surfaces, which families are clefined by invariant systems of partial differential equatious.

If a surface admits of no transformation of a $G_{n}$, it will generate an invariant family of $\infty^{n}$ surfaces, when subjected to all the transformations of the $G_{n}$. If this family of $\sigma^{n}$ surfaces is given by the equation

$$
z=f(x, y) \text {, }
$$

where $f$ is an analytical function, we can always write this equation in the form

$$
\begin{aligned}
z-z_{0}=p_{0}\left(x-x_{0}\right)+ & \frac{p_{0}}{2}\left(x-x_{0}\right)^{2}+s_{0}\left(x-x_{0}\right)\left(y-y_{0}\right) \\
& +\frac{t_{0}}{2}\left(y-y_{0}\right)^{2}+\ldots,
\end{aligned}
$$

where $z_{0}, p_{0}, \ldots, t_{0}, \ldots$ are the values of $z, p, \ldots, t, \ldots$, when we assign to $x$ and $y$ their initial values $x_{0}$ and $y_{0^{\circ}}$

Since the family consists of $\omega^{n}$ surfaces, $n$ of the arbitrary constants $z_{0}$, $p_{0}, \ldots, t_{10}, \ldots$ must be connected by no relations, while the remaining arbitrary constunts must be so connected by relations that they can be expressed in terms of the $n$ arbitrary constants which are connected by no relitions; that is, $n$ and only $n$ of the partial differential coefficients are comnected by no relations.

Now suppose that we have an invariant system of partial clifferential equa- 
tions, and that by means of these equations we can express all, except $n$ of the partial differential coefficients of $z$ with respect to $x$ and $y$ in terms of the remaining $n$ partial differential coefficients; it is clear that such a system of partial differential equations will defiue m invariant finnily of exactly on surfrces, since, in this case, $n$ of the arbitrary constants of equation (2) will be connected by no relation.

The system must cousist of an infinite number of partial differential equatious and it must be completely integrable.*. For equation (2) contains an intinite number of arbitrury constants, $z_{v}, p_{0}, \ldots, t_{0}, \ldots$ and all of these constints, except $n$, are determined by means of the partial differential equations in terms of the remaining $n$ arbitrary constants.

Since the system must contain an infinite number of equations, we can suppose the system has been so arranged that by differentiating one of the partial differential equations of the system, we ouly obtain another partial differential equation of the system already given.

We can also suppose that the equatious of the system are so arranged that beginning with the lowest order they proceed to those of higher orders ; and, finally, that from the equations of the $p$ th order we cannot eliminate all the partial differential coefficients of the $p$ th order. It is evident, then, that such a system of partial difierential equations as we have defined above will determine, from a certain point on, all the partial differential coefficients of $z$ with respect to $x$ and $y$ in terms of those of lower orders.

If the surface admits of $m$ of the indepeudent infinitesimal transformations of the $G_{n}$, where $m<n$, it will generate a family of $\omega^{n-m}$ surfaces. What we have said above in regard to the family of $\infty^{n}$ surfaces is equally true of this family of $\infty^{n-m}$; that is, it will be defined by a completely integrable invariant system consisting of an infinite number of partial differential equations, by means of which we can cletermine all the higher partial clifferential coefficients of $z$ with respect to $x$ and $y$ in terms of $(n-m)$ of the lower partial differential coefficients of $z$ with respect to $x$ and $y$.

36. We shall now show what invariant systems of partial differential equations are necessary to define the invariant finmilies of surfaces, which are composed of those surfaces that are equivalent by means of the transformations of the Gromp

$$
\frac{\partial f}{\partial x}, \frac{\partial f}{\partial y}, \frac{\partial f}{\partial z}, x \frac{\partial f^{\prime}}{\partial \alpha}, y \frac{\partial f}{\partial y}, z \frac{\partial f}{\partial z}, x^{2} \frac{\partial f}{\partial x}, y^{2} \frac{\partial f}{\partial y}, z^{2} \frac{\partial f}{\partial z} \text {. }
$$

* Golursat, p. 41, Vol. II. 
I. The invariant systems that contain no partial differential equation of an order lower than the third.

a. Suppose the invariant system contains one partial differential equation of the third order of the form (cf. $\$ 33, \mathrm{~d}$ )

$$
Q_{1}\left(\varphi_{1}, \varphi_{2}, \varphi_{3}\right)=0 \text {. }
$$

By means of this equation we can determine one of the partial differential coefficients of the third order in terms of the three remaiving partial differential coefticients of the third order and those 'of lower orders; that is, in terms of uine of the partial differential coefficients. Now, since the greatest family of surfaces invariant under the $G_{9}$ consists of $\infty$ "surfaces, it is evident that the greatest number of partial differential coefficients, that can be connected by $n 0$ relations, is nine; so that we must have, in connection with equation (3), other equations by means of which we can determine all the higher partial differential coefficients from the fourth order on in terms of the nine partial differential coefficients of lower orders (cf. p. 65). If we differentiate equation (3) partially with respect to $x$ and $y$, we have the two equations of the fourth order

$$
\frac{\partial Q_{1}}{\partial x}=0, \quad \frac{\partial \varrho_{1}}{\partial y}=0
$$

which must evidently form a system which is invariatut under the $G_{y}$. These two equations will determine two of the partial differential coefficients of the fourth order in terms of the nine of lower orders. In order to determine the three remaining partial differential coefficients of the fourth order, we must have three invariant equations of the fourth order (cf. $33 \mathrm{e}$ )

$$
\left.\begin{array}{l}
\varrho_{2}\left(\varphi_{1}, \ldots, \psi_{1}, \ldots, \phi_{5}\right)=0, \\
\varrho_{3}\left(\varphi_{1}, \ldots, \psi_{1}, \ldots, \phi_{5}\right)=0, \\
\varrho_{4}\left(\varphi_{1}, \ldots, \phi_{1}, \ldots, \phi_{5}\right)=0,
\end{array}\right\}
$$

no one of which can be a consequence of equation (3). By means of equations (3), (4), and (5), we can express one partial differential coefficient of the third order and the five partial differential coefficients of the fourth order in terms of the three remaining partial differential coefficients of the third order and the partial differential coefficients of lower orders.

Now by repeated partial differentiation of the above equations we obtain an infinite number of equations, belonging to a system which is invariant under the $G_{9}$. It is clear that by means of this system of equations we can determine all, except nine, of the partial differential coefficients of $z$ with respect to $x$ and $y$ in terms of nine of the partial differential coefticients; hence, in 
this case, nine and only nine of the arbitrary constants of equation (2) are connected by no relations, and we see, then, that the invariant system containing one partial aifferential equation of the thircl order and three of the fourth order will define an invariant fumily of exactly $\infty^{9}$ surfaces.

As a similar discussion holds for all the other cases, we need only write down the remaining invariant systems.

b. If the invariant system contains two independent partial differential equations of the third order,

$$
\begin{aligned}
& \Omega_{1}\left(\varphi_{1}, \varphi_{2}, \varphi_{3}\right)=0, \\
& \Omega_{2}\left(\varphi_{1}, \varphi_{2}, \varphi_{3}\right)=0,
\end{aligned}
$$

it will define an invariant family of exactly $\infty^{9}$ surfaces.

c. If the invariant system contains two partial differential equations of the third order,

$$
\begin{aligned}
& \Omega_{1}\left(\varphi_{1}, \varphi_{2}, \varphi_{3}\right)=0, \\
& \Omega_{2}\left(\varphi_{1}, \varphi_{2}, \varphi_{3}\right)=0,
\end{aligned}
$$

aud one partial differential equation of the fourth order,

$$
\Omega_{3}\left(\varphi_{1}, \ldots, \phi_{1}, \ldots, \phi_{n}\right)=0,
$$

which is not a consequence of those of the third order, it will define an invariant family of exactly $\infty^{3}$ surfaces.

d. If the invariant system contains three independent partial differential equations of the third order,

$$
\begin{aligned}
& \Omega_{1}\left(\varphi_{1}, \varphi_{2}, \varphi_{3}\right)=0, \\
& \Omega_{2}\left(\varphi_{1}, \varphi_{2}, \varphi_{3}\right)=0, \\
& \Omega_{3}\left(\varphi_{1}, \varphi_{2}, \varphi_{3}\right)=0,
\end{aligned}
$$

it will define an invariant family of exactly $\infty^{7}$ surfaces.

e. If the invariant system contains four independent partial differential equations of the third order,

$$
\begin{aligned}
& \Omega_{1}\left(\varphi_{1}, \varphi_{1}, \varphi_{3}\right)=0, \\
& \Omega_{2}\left(\varphi_{1}, \varphi_{2}, \varphi_{3}\right)=0, \\
& \Omega_{3}\left(\varphi_{1}, \varphi_{3}, \varphi_{3}\right)=0, \\
& \left.\left.\Omega_{4}\right) \varphi_{1}, \varphi_{2}, \varphi_{3}\right)=0,
\end{aligned}
$$

it will define an invariant family of exactly $\infty$ surfaces.

II. The invariant systems which contain partial differential equations of the second order and none of the first order. 
Since there are no partial differential equations of the second order invariant under the $G_{9}$, except the system $r=s=t=0$, which is a consequence of the invariant system of the first order $p=q=0$, there is no invariant system containing a partial differential equation of the second order and none of the first order. (Cf. 33, c.)

III. The invariant systems which contain partial differential equations of the first order.

a. If the invariant system contains the two partial differential equations of the first order, of the typical forms

$$
\begin{aligned}
& p=0, \\
& q=0,
\end{aligned}
$$

it will define an invariant family of $\infty^{1}$ surfaces. Evidently, in this case, the invariant family consists of the planes

$$
z=\text { const. }
$$

b. If the invariant system contains one partial differential equation of the first order and one of the third order, it will define an invariant family of exactly $\infty^{3}$ surfaces.

On account of the symmetry of the $G_{n}$, we may choose

$$
p=0
$$

as the typical partial clifferential equation of the first order ; and, then, it is clear that the partial differential equation of the third order cannot be a consequence of $p=0$.

If we put

$$
\varphi_{1} \equiv \frac{q^{1}\left(3 r^{2}-2 p \pi\right)}{p^{4}\left(3 t^{2}-2 \eta \tau\right)}
$$

equal to infinity, we obtain the partial differential equation of the third order

$$
3 t^{2}-2 \eta \tau=0
$$

This is the only invariant partial differential equation of the third order that is not a consequence of $p=0$, so that the invariant system is

$$
\begin{gathered}
p=0 \\
3 t^{2}-2 q \tau=0 .
\end{gathered}
$$

When $p=0, z=F(y)$, and the equation $3 t^{2}-2 q \tau=0$ may be written .

$$
3\left[\frac{d^{2} z}{d y^{2}}\right]^{2}-2 \frac{d z}{d y} \frac{d^{3} z}{d y^{3}}=0
$$

The integral of this equation is (cf. $\$ 27$ )

$$
y z+\alpha z+\beta y+\gamma=0 \text {. }
$$

Hence, in this case, the invariant family consists of the $\infty^{3}$ hyperbolic cylinders given by the above equation. 
ROBERIS. ON THE TRANSFOTMATION GROUP, E'TC.

There are no other systems containing a partial differential of the first order invariant under the $G_{0}$, for there are no invariant partial differential equations of the fourth or higher orders that are not a consequence of $p=0$.

37. We may now collect our results as follows:

A. If the invariant family consists of $\infty^{9}$ surfaces, it will be defined by

1. An invariant system containing one partial differential equation of the third order and three of the fourth order, or

2. An invariant system containing two partial differential equations of the third order.

B. If the invariant family consists of $\infty^{s}$ surfaces, it will be defined by an invariant system containing two partial differential equations of the third order and one of the fourth order.

C. If the invariant family consists of $\infty^{7}$ surfaces, it will be defined by an invariant system containing three partial differential equations of the third order.

D. If the iuvariant family consists of $\infty^{6}$ surfaces, it will be defined by an invariant system containing four partial differential equations of the third order.

E. There are no invariant systems that will define invariant families, consisting of $\infty^{5}, \infty^{4}$, or $\infty^{2}$ surfaces ; hence no surface admits of exactly four, five, or seven independent infinitesimal transformations of the $G_{19}$.

F.. If the invariant family consists of $\infty^{3}$ surfaces, it will be defined by an invariant system containing one partial differential of the first order and one of the third.

G. If the invariant family consists of $\infty^{1}$ surfaces, it will be defined by an invariant system containing two partial differential equations of the first order.

In this, the last section of this paper, we have shown how we may determine the nature of the invariant families of surfaces, which are composed of those surfuces that are equivalent by means of the transformations of the $G_{0}$; and if we have two surfaces that are equivalent by means of the transformations of the Group, their equations must satisfy the partial differential equations of some one of the invariant systems enumerated in cases I to III.

In the discussion of the above group I have followed the method of Sophus Lie, which can be found in bis Vorlesungen ueber Continuierliche Gruppen: Where it has been cleemed advisable I have given special references, 